\title{
Short-horizon excess returns and exchange rate and interest rate effects
}

\author{
Nathan Lael Joseph ${ }^{\mathrm{a}}$, Neophytos Lambertides ${ }^{\mathrm{b}}$, Christos S. Savva ${ }^{\mathrm{c}}$
}

a Nathan Lael Joseph, Aston Business School, Aston University, Birmingham, B4 7ET, United Kingdom; tel. 44-121-204-3143; email n.1.joseph@aston.ac.uk.

${ }^{\mathrm{b}}$ Neophytos Lambertides (corresponding author), Cyprus University of Technology, Department of Commerce, Finance and Shipping, 3603 Lemesos, Cyprus; tel. +357 25 002591; email n.lambertides@cut.ac.cy.

c Christos S. Savva, Cyprus University of Technology, Department of Commerce, Finance and Shipping, 3603 Lemesos, Cyprus; tel. +357 25 002349; email christos.savva@cut.ac.cy. 


\title{
Short-horizon excess returns and exchange rate and interest rate effects
}

\begin{abstract}
We examine the effects of foreign exchange (FX) and interest rate changes on the excess returns of U.S. stocks, for short-horizons of 1 to 40 days. Our new evidence shows a tendency for the volatility of both excess returns and FX rate changes to be negatively related with FX rate and interest rate effects. Both the number of firms with significant FX rate and interest rate effects and the magnitude of their exposures increase with the length of the return horizon. Our finding seems inconsistent with the view that firms hedge effectively at short-return horizons.
\end{abstract}

JEL: F3; G15

Keywords: Exchange rate and interest rate effects; smooth transition function; bivariate GJR-GARCH-M; time-varying conditional correlations; Fama-French-Carhart (FFC) factors 


\section{Introduction}

Empirical studies that focus on the effects that foreign exchange (FX) rate and interest rate changes have on stock returns, primarily employ one-period returns. Despite the theoretical predictions (Shapiro, 1974; Marston, 2001), there is very little empirical support for the view that FX rate and interest rate changes affect the returns of non-financial stocks when one-period returns are used (Jorion, 1990; Bartov and Bodnar, 1994; Choi and Prasad, 1995; Bartram, 2002). Prior studies show that typically between 5\% and 29\% of U.S. firms exhibit significant FX rate effects (e.g., Jorion, 1990; Muller and Verschoor, 2008). ${ }^{1}$ This result holds across different countries as well as across non-financial industry sector portfolios. ${ }^{2}$ Interest rate effects are also weak for both non-financial stocks and non-financial industry sector portfolios (see Ehrhardt, 1991; Prasad and Rajan, 1995). Neither theory nor empirical work provides guidance about the proportion of firms that should exhibit significant FX rate and interest rate exposures. However, in theory, all firms are affected by FX rate and interest rate changes since parity conditions do not hold, except in the long-run.

Long-horizon return studies provide stronger support for FX rate effects for both U.S. and non-U.S. nonfinancial firms (Chow et al., 1997a, 1997b; Dominguez and Tesar, 2006). Between 60\% and 67\% of European and U.S. non-financial firms exhibit significant FX rate effects when long-horizon returns are used (Bodnar and Wong, 2003; Muller and Verschoor, 2006b). U.S. firms also experience default and term premia effects that increase with the length of the return horizon (see Chow et al., 1997a). Despite these findings, recent U.S. studies show that risk management leads to real effects on the value of firms-their market risk, leverage and their liquidity (Chen, 2011; Cornaggia, 2013). As such, Pérez-González and Yun (2013) show the market-to-book value of U.S. firms that use weather derivatives increases by at least $6 \%$ - a result that seems at odds with those of earlier related work (see Jin and Jorion, 2006). Even if risk management has effects on firm value, there seems to be a relatively large number of firms with residual exposure when long-horizon returns are estimated.

To date, no empirical study has examined the sensitivity of short-horizon stock returns to FX rate and interest rate changes, except with regard to one-period daily, weekly or monthly returns (see, e.g., Jorion, 1990; He and $\mathrm{Ng}, 1998$ ). For long-horizon studies the highest return frequency is weekly (see Muller and Verschoor, 2008) whereas, most studies use monthly returns (see Chow et al., 1997a). We use daily returns and return horizons of 1 to 40 days as this allows us to analyze short-horizon returns at a higher return

\footnotetext{
${ }^{1}$ Using a 5\% significance level, Jorion (1990) finds that at most, $5.6 \%$ of the 287 non-financial U.S. firms in his sample are significantly impacted by FX rate changes whereas, Muller and Verschoor (2006a) find that $29 \%$ of the 935 non-financial U.S. firms in their sample are significantly impacted by FX rate changes.

${ }^{2} \mathrm{He}$ and $\mathrm{Ng}$ (1998) find that $26 \%$ of non-financial Japanese firms exhibit significant FX rate effects. Similarly, Prasad and Rajan (1995) find that $25 \%$ of U.S. industry portfolios exhibit significant FX rate effects.
} 
frequency compared to prior work. We focus on short-horizon returns since it is often argued that firms are very effective in managing and hedging their short-term exposures (see Booth, 1996; Chow et al., 1997a, 1997b). We assume that our return horizons fall within the time period suggested by prior studies. $^{3}$ The idea that firms have good hedging skills is often used to explain why one-period returns provide very weak evidence of FX rate and interest rate effects. This explanation seems reasonable since Fortune 500 firms hedge their cash flow exposures over a two-to-four-quarter horizon (see Dolde, 1993), and up to $82 \%$ of U.S. firms hedge for an initial maturity of 90 days or less (see Bodnar et al., 1998, pp. 7577). So the explanation sits well when linked with risk management practice. Correspondingly, long-horizon returns appear to provide stronger evidence of exposure effects relative to one-period returns, due to the increased difficulty firms have in capturing long-term economic exposure (see Booth, 1996). Of course, CAPM estimates of exposure represent residual risk to both firms and investors.

We also examine the conditional correlations obtained from the volatility of excess stock returns and the volatility of FX rate changes. This is because the conditional volatility of excess returns and FX rate changes can affect the relation between excess returns and FX rate changes. There is growing support for this view. Bartov et al. (1996), for example, suggest that the volatility of stock returns appears to increase with the volatility of FX rate changes. We statistically test this view using two separate bivariate (asymmetric) GJRGARCH(1,1) in mean, hereafter, GJR-GARCH-M, that incorporates CAPM. ${ }^{4}$ Volatility spillover can also arise across the stock and FX markets to affect the relation between excess returns and FX rate changes (see Kanas, 2000). So large positive or negative shocks can increase the conditional correlation associated with the volatility of excess returns and FX rates changes in the same way that such shocks affect international stock markets (see, e.g., Longin and Solnik, 2001). While we do not specifically seek to explain the FX rate puzzle which has largely been done by others (see Booth, 1996; Miller and Reuer, 1998), we provide evidence on statistical patterns that have implications for the results of prior work.

As stated earlier, we estimate a bivariate GJR-GARCH-M in two separate augmented versions of CAPM. A complication of our bivariate models compared to exposure models used for long-horizon studies is that we estimate sensitivity in terms of the first lag of FX rate and interest rate changes. This approach is necessitated by our econometric methodology (see Section 3). Even so, we also run a univariate version

\footnotetext{
${ }^{3}$ The finance literature seems to consider returns taken at daily and/or weekly intervals to be at short-horizons, whereas, monthly and more distant returns appear to be considered to be returns at long horizons. In long-horizon studies, where typically one-period monthly returns are used, the length of the return horizon increases to up to 60 overlapping months (see Chow et al., 1997b). We consider our returns to be at short return horizons since we use daily returns and increase the length of the return horizon to up to 40 non-overlapping days-a constraint imposed by our use of non-overlapping returns (see Section 5.2).

${ }^{4}$ The GJR-GARCH-M model is the formulation of the asymmetric GARCH-M method of Glosten et al. (1993) following on from prior ARCH-M and GARCH-M processes. GARCH-type estimation methods generate more efficient coefficient estimates compared to standard ordinary least squares (OLS) and do not require corrections for autocorrelation and heteroscedasticity as done in prior work (e.g., Bartov et al., 1996; Chow et al., 1997a) that use OLS-based methods.
} 
of our three-factor CAPM using contemporaneous FX rate and interest rate changes and we find results that can be considered to be comparable to those we present (see Section 3).

Our main findings are as follows. Under the constant conditional correlation assumption, we find that $16.78 \%(20.72 \%)$ of U.S. firms exhibit significant (lagged) FX rate (interest rate) effects for one-period (daily) returns using our three-factor CAPM. Our six-factor CAPM which incorporates the Fama and French (1992; 1993) SMB and HML pricing factors and the Carhart (1997) momentum (MOM) factor, generates weaker support for FX rate (15.08\%) and interest rate (17.13\%) effects. This latter result is expected since the pricing factors, hereafter, FFC, are likely to capture macroeconomic effects (see Simpson and Ramchander, 2008) that perhaps, reduce FX rate and interest rate effects.

The number of firms with significant FX rate and interest rate effects increases substantially with the length of the return horizon in line with prior work, as does, the magnitude of their sensitivity coefficients. From day 1 to day 40, the percentage increase in the number of firms with significant FX rate effects is $61.27 \%$ under the three-factor CAPM. For the six-factor CAPM, the percentage increase is $126.63 \%$. We find similar increases in interest rate effects. The percentage increase in FX rate effects from day 1 to day 2 is $14.71 \%$ (29.35\%) under the three-factor (six-factor) CAPM. No horizon-on-horizon increases exceed this percentage, except for day 17 and day 30 for the three-factor CAPM and day 20 for the six-factor CAPM. ${ }^{5}$ In contrast, the percentage increase in interest rate effects from day 1 to day 2 is $80.16 \%$ (112.44\%) for the three-factor (six-factor) CAPM. At day 40, 27.06\% of the individual firms in our full sample exhibit significant FX rate effects under the three-factor CAPM compared with $34.18 \%$ for our six-factor CAPM. These percentages are only slightly better than those of long-horizon studies if we interpolate eight weeks (40 days) from the return horizons used by Bodnar and Wong (2003) and Muller and Verschoor (2006b). While Chow et al. (1997b, p. 198) show that the magnitude of the FX rate coefficients becomes significant at return horizons of 12 months or longer, we find that the increase in FX rate effects occurs much earlier when we examine both the frequency of FX rate effects and the magnitude of the exposure coefficients. So, the view that one-period returns provide weak support for exposure effects because U.S. firms are more effective when hedging short-term exposures (see Chow et al., 1997a; 1997b; Muller and Verschoor, $2006 \mathrm{~b}$ ) is not entirely in line with our findings. This is because we consider 40 days or even 2 days to be very short to obtain significant exposure effects. Indeed, Fehle and Tsyplakov's (2005) theoretical model shows that the risk management and hedging activities of firms do not depend on the automatic replacement

\footnotetext{
${ }^{5}$ To explore this result, we consider Bodnar and Wong's (2003, p. 43) findings, where $d$-period return horizons of 1, 3, 6, 9, 12, 18, ..., 36, 48 and 60 months are used. The intervals are not constant for $d$-periods beyond $d=12$ months. This does not affect the argument we wish to make. Their results show that the percentage horizon-on-horizon increases in FX rate effects, i.e., $d=1$ to $3, d=3$ to 6 months, and so on, are respectively, $12.7 \%,-5.9 \%,-6.6 \%,-0.4 \%, 1.7 \% \ldots, 11.5 \%$, and $8.7 \%$. Clearly, the largest percentage increase in FX rate effects is at the earliest return horizons of $d=1$ to 3 months with a declining rate of increase after $d=12$ months. This is in line with our findings as well as Muller and Verschoor's (2008b) findings, where weekly returns are used.
} 
of expiring contracts but, rather, on the level of firms' product prices, their financial characteristics, and hedges already in place. So, substantial exposure can arise at short-return horizons contrary to the implications of prior work.

At day 1 , roughly $44 \%$ of the conditional correlations associated with the volatility of excess returns and FX rate changes are significant. This percentage decreases as the return horizon increases, especially at the earliest return horizons. The largest percentage decrease in the number of significant conditional correlations is from day 1 to day 2, exactly when the number of firms with significant FX rate and interest rate effects exhibits the highest percentage increase. Similarly, the number of firms with significance conditional volatility and asymmetry coefficients decreases as the return horizons increase. This latter finding reflects the view that volatility has less impacts on more distant expected returns. We find comparable results for our smooth transition conditional correlation (STCC) although here, a smaller number of firms exhibits significant FX rate and interest rate effects compared with the constant conditional correlation case.

The remaining sections of this study are organized as follows. Section 2 presents a review of prior work. Section 3 presents the empirical models used in our study and Section 4 presents the data sets. Section 5 discusses our empirical results and Section 6 concludes the study.

\section{Background}

Several explanations have been put forward for the failure of CAPM-based approaches to provide strong evidence of FX rate effects. One explanation suggests that FX rate effects are likely to be reduced since firms hedge their corporate exposures (see Booth, 1996). This argument also applies to interest rate effects. However, Pantzalis et al. (2001) show that after eliminating firms that do not use financial hedges, the percentage of U.S. firms that are exposed to FX rate changes falls to between $0 \%$ and $6 \%$, depending on the choice of both the FX rate index and the stock index used to capture the overall market return. Also, the greater the geographical spread of firms and their global involvement, the more scope firms have to offset their exposures, thereby minimizing the predicted exposure effect. Pricing-to-market strategies and the exercise of strategic options are also argued to reduce FX rate effects (Booth, 1996; Miller and Reuer, 1998).

Attempts to control for the factors that are claimed to limit the predicted relation do not always improve on existing results. Amihud (1994), for example, uses a sample of non-financial firms from the Fortune list of 50 Leading Exporters and still finds weak evidence of FX rate effects at both the firm and aggregate portfolio levels. Also, Muller and Verschoor (2008) show that U.S. firms that concentrate in Latin American countries are more exposed to FX rate changes than those that are more geographically spread out. U.S. 
firms with more operating networks (breadth) and more foreign subsidiaries (depth) are also more exposed to FX rate changes (see Pantzalis et al., 2001). Even for the more opened economies, such as Japan, the percentage of non-financial firms with FX rate effects is still low (see $\mathrm{He}$ and $\mathrm{Ng}, 1998$ ).

Mis-measurement also affects the predicted relation. Bodnar and Wong (2003) suggest that the use of valueweighted market returns as opposed to equally-weighted market returns is more likely to bias the results toward little or no exposure. This is because value-weighted returns favor large firms more than small firms and, since large firms also have more breadth and depth because of size, FX rate effects will be lower both in frequency and magnitude. Dominguez and Tesar (2006) find limited empirical support for the view regarding the frequency of FX rate exposure effects. The choice of the bilateral FX rate or trade-weighted FX rate index produces only small improvements in the results (see Muller and Verschoor, 2008).

Following Chow et al. (1997a, 1997b), several empirical studies show that the number of firms with significant FX rate effects increases with the length of the return horizon (Bodnar and Wong, 2003; Dominguez and Tsar, 2006; Muller and Verschoor, 2006a). These studies suggest that long return horizons generate stronger support for FX rate effects because firms hedge less perfectly at long return horizons. This general result is not too surprising if in the long-run most economic variables reach some equilibrium level. In the Sharpe-Lintner CAPM, stock returns are assumed to be linearly related to market returns such that the market beta captures the cross-sectional variation in expected average returns. Average stock returns are related to size and the ratio of book-to-market equity (see, e.g., Banz, 1981). Short-term stock returns also contain momentum (Jegadeesh and Titman, 1993). Fama and French (1992; 1993) show that size and book-to-market effects are related and can be captured by: i) the difference in the returns of a portfolio based on small stocks and another portfolio based on large stocks (SMB); and, ii) the difference between a portfolio based on high book-to-market stocks and another portfolio based on low book-to-market stocks (HML). Carhart (1997) shows that the difference between the average returns of two previously highreturn portfolios and the average returns of two previously low-return portfolios can capture the momentum (MOM). Since SMB and HML are considered to correct for underestimated market betas (Ferguson and Shockley, 2003) and MOM corrects for investor irrationality and/or over/under-reaction, their use will improve model specification. So we also estimate a bivariate GJR-GARCH-M that contains a CAPM that incorporates the FFC factors to correct for mis-pricing effects. ${ }^{6}$

\footnotetext{
${ }^{6}$ We note, however, that Fraser and Pantzalis (2004) mention in a footnote that they find no difference in their results for FX rate effects when SMB and HML are incorporated in their model (see also Bodnar and Wong, 2003). Du and Hu (2012) also report no difference in their results for FX rate volatility when the FFC pricing factors are included in their model. Unlike Fraser and Pantzalis (2004) and Du and $\mathrm{Hu}$ (2012), we employ bivariate GJR-GARCH-M methods where the conditional correlations are either held constant or allowed to vary. Our CAPMs also incorporate interest rate changes and we estimate two augmented CAPMs, one of which contains FFC pricing factors.
} 


\section{Econometric specification}

In each bivariate GJR-GARCH-M specification, there are two mean and two variance equations. The mean equations in our bivariate GJR-GARCH-M version of the three-factor CAPM (stock return equation) and FX rate equation are, respectively,

$$
\begin{gathered}
Z_{i, t}=\alpha_{1, i}+\beta_{1, m, i} Z_{m, t}+\lambda_{1, F X, i} R_{F X, t-1}+\gamma_{1, f, i} R_{f, t-1}+\xi_{1, i} D_{t}+\delta_{1, i} h_{1, t}^{2}+\varepsilon_{1, i, t} \\
R_{F X, t}=\alpha_{2, i}+\lambda_{2, F X, i} R_{F X, t-1}+\varepsilon_{2, i, t}
\end{gathered}
$$

where $Z_{i, t}=\left(R_{i, t}-r_{f, t}\right)$ is the excess daily return for stock $i . R_{i, t}=\left(r_{i, t}-r_{i, t-1}\right) / r_{i, t-1}$ is the stock return and $r_{f, t}$ is the risk-free rate. $Z_{m, t}=\left(R_{m, t}-r_{f, t}\right)$ is the excess return on the value-weighted overall market index where $R_{m, t}=\left(r_{m, t}-r_{m, t-1}\right) / r_{m, t-1} . R_{F X, t}=\left(r_{F X, t}-r_{F X, t-1}\right) / r_{F X, t-1}$ denotes the return/change for the FX rate index. $R_{f, t}$ is the change in the risk-free interest rate to capture interest rate effects. We estimate $R_{f, t}$ in Eq. (1a) with a lag to be consistent in time with $R_{F X, t-1}$. To estimate the bivariate specification, $R_{F X, t}$ must appear in Eq. (1a) with a lag since we need to have the contemporaneous FX rate change, $R_{F X, t}$ in Eq. (1b). Otherwise, the bivariate model cannot be estimated. The trade-off of not being able to capture contemporaneous FX rate and interest rate effects in Eq. (1a) can be considered to be off-set by the ability of our bivariate model to simultaneously estimate the conditional correlations associated with Eqs. (1a) and (1b). While the exposures we estimate here may be considered to be model specific, we test for variation in our results against a univariate version of equation Eq. (1a) that incorporates contemporaneous FX rate and interest rate changes as right-hand-side variables. ${ }^{7}$ The results of this univariate equation and the bivariate case can be considered to be comparable. ${ }^{8}$ Following on from Eq. (1a), $D_{t}$ is a dummy variable that captures the effects of the recent financial crisis on excess stock returns. $D_{t}$ takes on a value of one for the period July 1, 2007 to June 30, 2009; zero otherwise. The FX

\footnotetext{
${ }^{7}$ Specifically, for one-period returns, 6.99\% of the firms in our full sample exhibit significant FX rate effects under our univariate threefactor CAPM compared with $16.78 \%$ for the bivariate case. The percentage for the univariate CAPM compares favorably with Jorion's (1990) results. For day 5 and day 40 return horizons, the results are $13.73 \%$ and $25.49 \%$ respectively for the univariate CAPM compared with $19.33 \%$ and $27.06 \%$ for the bivariate case. For interest rate effects under the univariate three-factor CAPM, the percentages are: $18.57 \%, 24.58 \%$ and $35.52 \%$ for day 1,5 and 40 return horizons, respectively. For the bivariate case the percentages are: $20.72 \%, 25.16$ and $36.02 \%$ for day 1,5 and 40 , respectively. Clearly, across the models, the results for interest rate effects are more comparable than those of FX rate effects. Overall, we take the view the univariate and bivariate specifications generally lead to more or less comparable results.

${ }^{8}$ Jayasinghe and Tsui (2008) also use $R_{F X, t-1}$ in their bivariate GJR-GARCH. Eqs. (1a) and (1b) captures the basic elements of their bivariate GJR-GARCH model for the raw returns of Japanese sector portfolios. The authors do not estimate the in-mean version of GJRGARCH and they also exclude interest rate changes and FFC pricing factors. Our specification, like theirs, does not allow for contemporaneous FX rate changes as a right-hand-side variable.
} 
rate index does not appear to be affected by the financial crisis in our statistical tests. $h_{1, t}^{2}$ is the (in-mean) conditional variance or risk tolerance measure, which theoretically, is linearly related to $Z_{i, t}$. $\varepsilon_{1, i, t}$ and $\varepsilon_{2, i, t}$ are a $2 \times 1$ vector of the pair-wise error terms for stock excess return $i$ and FX rate changes. The conditional errors of our parameter estimates are assumed to follow a bivariate normal distribution with zero mean and unit variance.

The bivariate GJR-GARCH-M for the mean equations of the six-factor CAPM (stock return equation) and $\mathrm{FX}$ rate equation are respectively,

$$
\begin{gathered}
Z_{i, t}=\alpha_{3, i}+\beta_{2, m, i} Z_{m, t}+\lambda_{3, F X, i} R_{F X, t-1}+\gamma_{2, f, i} R_{f, t-1}+\xi_{2, i} D_{t}+\delta_{2, i} h_{2, t}^{2}+\eta_{1, i} S M B_{t} \\
+\eta_{2, i} H M L_{t}+\eta_{3, i} M O M_{t}+\varepsilon_{3, i, t}
\end{gathered}
$$

$S M B_{t}, H M L_{t}$, and $M O M_{t}$ are as described in Section 2. The definitions for the remaining variables are similar to those in Eqs. (1a) and (1b).

The conditional variance equations for Eqs. (1a) and (1b) are respectively,

$$
\begin{aligned}
& \varepsilon_{1, i, t} \mid \Omega_{1, i, t-1}=h_{1, i, t}^{2}=\mu_{1, i}+\phi_{1, i} \varepsilon_{1, i, t-1}^{2}+\varphi_{1, i} h_{1, i, t-1}^{2}+\vartheta_{1, i} S_{1, i, t-1}^{2} \varepsilon_{1, i, t-1}^{2}+\xi_{3, i} D_{t} \\
& \varepsilon_{2, i, t} \mid \Omega_{2, i, t-1}=h_{2, i, t}^{2}=\mu_{2, i}+\phi_{2, i} \varepsilon_{2, i, t-1}^{2}+\varphi_{2, i} h_{2, i, t-1}^{2}+\vartheta_{2, i} S_{2, i, t-1}^{2} \varepsilon_{2, i, t-1}^{2}
\end{aligned}
$$

Here, $\varepsilon_{1, i, t} \mid \Omega_{1, t-1}$ and $\varepsilon_{2, i, t} \mid \Omega_{2, t-1}$ denote the conditional errors of Eqs. (1a) and (1b) respectively, based on past information. $\mu_{1, i}$ and $\mu_{2, i}$, respectively, capture the long-term conditional variance (permanent component) of stock excess returns and FX rate changes. Similarly, $\phi_{1, i}$ and $\phi_{2, i}$ capture the impact of past news in each equation, while $\varphi_{1, i}$ and $\varphi_{2, i}$ capture the impact of past volatility on current volatility. $\vartheta_{1, i}$ and $\vartheta_{2, i}$ are respectively the coefficients for asymmetric effects - leverage effects. They are based on dummy variables $S_{1, i, t-1}^{2}$ and $S_{2, i, t-1}^{2}$ that carry a value of one when $\varepsilon_{1, i, t-1}$ and $\varepsilon_{2, i, t-1}$ are respectively negative, otherwise zero. Eq. (3b) contains a coefficient for conditional asymmetry since FX rate changes have been shown to exhibit asymmetric effects (see Chkili, et al., 2012). $\xi_{3, i}$ denotes the coefficient for the financial crisis over the same period as for stock returns.

Similarly, the conditional variance equations for Eqs. (2a) and (2b) are respectively, 


$$
\begin{gathered}
\varepsilon_{3, i, t} \mid \Omega_{3, i, t-1}=h_{3, i, t}^{2}=\mu_{3, i}+\phi_{3, i} \varepsilon_{1, i, t-1}^{2}+\varphi_{3, i} h_{3, i, t-1}^{2}+\vartheta_{3, i} S_{3, i, t-1}^{2} \varepsilon_{3, i, t-1}^{2}+\xi_{4, i} D_{t} \\
\varepsilon_{4, i, t} \mid \Omega_{4, i, t-1}=h_{4, i, t}^{2}=\mu_{4, i}+\phi_{4, i} \varepsilon_{4, i, t-1}^{2}+\varphi_{4, i} h_{4, i, t-1}^{2}+\vartheta_{4, i} S_{4, i, t-1}^{2} \varepsilon_{4, i, t-1}^{2}
\end{gathered}
$$

The definitions for the coefficients in Eqs. (4a) and (4b) are similar to those for Eqs. (3a) and (3b).

The bivariate GJR-GJR-GARCH-M specifications are first estimated using Bollerslev's (1990) approach, where the variances and covariances are time varying but the conditional correlations are constant. We use the results of the constant conditional correlation as a benchmark against which we compare the results of the STCC-GJR-GARCH-M where the conditional correlations are allowed to vary (see Silvennoinen and Teräsvirta, 2005). Following Bollerslev (1990), the time-varying conditional covariances are assumed to be proportional to the square of the product of both conditional variances in the bivariate equations. Thus, for Eqs. (1a) and (1b), the variance associated with the conditional correlation can be stated as,

$$
h_{12, t}=\rho_{12}\left(h_{1, i, t}, h_{2, i, t}\right)^{1 / 2}
$$

where $\rho_{12}$ is the conditional correlation. Similarly, the variance associated with the conditional correlation for Eqs. (3a) and (3b) can be stated as,

$$
h_{34, t}=\rho_{34}\left(h_{3, i, t}, h_{4, i, t}\right)^{1 / 2}
$$

where $\rho_{34}$ is the conditional correlation. Estimates for the mean equations, the conditional variances and conditional correlations are estimated jointly, leading to efficiency gains in estimation.

As stated before, the conditional correlations are allowed to vary under the STCC-GJR-GARCH-M (see Silvennoinen and Teräsvirta, 2005). We estimate the STCC-GJR-GARCH-M as opposed to the dynamic conditional correlation (DCC)-GJR-GARCH-M, since unlike the DCC-GJR-GARCH-M, the STCCGJR-GARCH-M generates a slope parameter that captures the rate of change in the conditional correlations. We want to estimate this parameter since it may help explain the observed increase in exposure effects as the length of the return horizon increases. Of course, the choice of the constant conditional correlation as opposed to the time-varying case is an empirical question.

To implement the STCC-GJR-GARCH-M, we must first test for a structural break in the excess stock returns of each firm, using the $\mathrm{LM}_{\mathrm{CC}}$ statistic (see below). Failure to reject the null hypothesis implies that the conditional correlations are constant. Otherwise, we proceed to estimate the STCC-GJR-GARCH-M 
by allowing the volatility of the conditional correlations to vary. The number of parameters to be specified is substantial. So we limit, where appropriate, the use of subscripts to describe the parameters for each bivariate model in a fairly general way. Thus for the bivariate model containing the three-factor CAPM, the time-varying conditional correlations are given by,

$$
\begin{aligned}
& h_{12, t}=\rho_{12, t}\left(h_{1, i, t}, h_{2, i, t}\right)^{1 / 2}, \\
& \rho_{12, t}=\rho_{12 a}\left(1-G\left(s_{i, t} ; c_{1}, g_{1}\right)\right)+\rho_{12 b} G\left(s_{i, t} ; c_{1}, g_{1}\right)
\end{aligned}
$$

where the parameters $\rho_{12 a}$ and $\rho_{12 b}$ denote the two extreme states or regimes for the conditional correlations of stock $i$. These extreme states are bounded between zero and one according to the continuous transition function that takes the general form $G\left(s_{i, t} ; c, g\right)$. This logistic transition function captures the temporal changes in the contemporaneous conditional correlation, $\rho_{12, t}$. The general form of the logistic transition function can be written as,

$$
G\left(s_{i, t} ; c, g\right)=\frac{1}{1+\exp \left(-g\left(s_{i, t}-c\right)\right)}, g>0
$$

where $s_{i, t}$ is the transition variable for stock $i$ (represented by $R_{F X, t}$ ), while parameters $c$ and $g$ capture respectively, the location and the smoothness coefficients for the transition function, for shifts between regimes $\rho_{12 a}$ and $\rho_{12 b}$. The transition function is bounded between zero and one, provided there are valid conditional correlations between -1 and +1 . We use a grid search method to establish the starting values of $c$ and $g$. Different values of $\rho_{12 a}$ and $\rho_{12 b}$ imply that the conditional correlations increase ( $\left.\rho_{12 a}<\rho_{12 b}\right)$ or decrease $\left(\rho_{12 a}>\rho_{12 b}\right)$ with the pace of change determined by $g$ in Eq. (7). The conditional correlation, $\rho_{12, t}$, is influenced by the transition variable in relation to the location parameter c. The parameter $c$ can be interpreted as the threshold between the two regimes corresponding to $1-G\left(s_{i, t} ; c_{1}, g_{1}\right)=0$ and $G\left(s_{i, t} ; c_{1}, g_{1}\right)=1$ in Eq. (6).

For the bivariate model containing the six-factor CAPM, the time-varying conditional correlations is given by

$$
\begin{aligned}
& h_{34, t}=\rho_{34, t}\left(h_{3, i, t}, h_{4, i, t}\right)^{1 / 2}, \\
& \rho_{34, t}=\rho_{34 a}\left(1-G\left(s_{i, t} ; c_{1}, g_{1}\right)\right)+\rho_{34 b} G\left(s_{i, t} ; c_{1}, g_{1}\right)
\end{aligned}
$$


The parameter definitions for Eq. (8) are similar to those of Eq. (6). The same transition function in Eq. (7) applies. Eqs. (6) to (8) are estimated using maximum likelihood function in one full step, for each bivariate model. 


\section{Dataset}

We use U.S. daily stock prices (inclusive of dividends) from the CRSP database for the period January 1, 2001 to December 31, 2010. All firms with fewer than 500 continuous trading days are deleted from our sample. We run our bivariate models for 1,220 U.S. non-financial firms. Our firms cover 10 industrial sectors. These industrial sectors reflect the 10 industry portfolio groups on Professor Kenneth French's website. ${ }^{9}$ The manufacturing sector contains the largest percentage of firms (17.33\%), while the Telephone and Television Transmission sector contains the smallest percentage of firms (2.73\%). The observations for $S M B_{t}, H M L_{t}$, and $M O M_{t}$ are taken from Professor Kenneth French's website. We use the trade-weighted U.S. dollar FX rate index based on a basket of currencies. The trade-weighted index helps avoid potential multicollinearity that can arise when several bilateral FX rates are used. Our riskfree rate is the U.S. three-month Treasury bill, deannualized for one day. Both the Treasury bill rate and the FX rate index are taken from DataStream.

\section{Empirical results}

This main section presents our empirical results. We first present the results for both CAPMs under the GJR-GARCH-M for day 1 returns under the constant conditional correlation assumption. We obtain coefficient estimates for 1,216 firms under the three-factor CAPM and 1,220 firms under the six-factor CAPM. This difference reflects the ability of our GJR-GARCH-M algorithm to reach convergence. We also relate the exposure effects to firm size. These results are presented in Section 5.1.2. Section 5.2 presents the results for the return horizons. Our $d$-period return horizons are $d=1,2,5,8,11,14,17,20,30$, and 40 days. Our use of non-overlapping returns limits the number of return horizons we can estimate. There is little theoretical guidance on what the lengths of the $d$-periods should be. We replicate these results in Section 5.3 for our STCC-GJR-GARCH-M bivariate models by allowing the conditional correlations to vary. We present the results for our conditional variances and conditional correlations in Section 5.4.

\subsection{Coefficients of mean equations under the constant conditional correlation for one-period returns}

Using Eqs. (1a) to (3b), firm-by-firm regressions are estimated under the constant conditional correlation assumption. We report the average coefficient by explanatory variable according to their sign to avoid the averaging-out effect of positive and negative coefficients by variable, when aggregation is used. Using the estimated coefficients as observations, we estimate the $t$-statistic to test the significance of the average coefficients. Like prior studies (see, e.g., Jorion, 1990; Muller and Verschoor, 2006a), we show the number

\footnotetext{
${ }^{9}$ See http://mba.tuck.dartmouth.edu/pages/faculty/ken.french/Data Library. We thank Professor Kenneth French for making the FFC pricing factors available.
} 
of firms with significant coefficients based on the sign of the coefficients. The average values for the coefficients based on their coefficient sign are also shown, where appropriate.

\subsubsection{Coefficients of mean equations}

This sub-section focuses on the results for one-period (day 1) returns to provide a basis for comparison with the longer horizon results in Section 5.2. Table 1 shows that the average coefficients are statistically significant $(p$-value $\leq 0.10)$ for both bivariate models. The average for all positive $\beta_{1, m, i}$ under the threefactor CAPM is 0.992 whereas, the average for positive $\beta_{2, m, i}$ under the six-factor CAPM is 0.967 . The difference between the beta averages is statistically significant, according the Mann-Whitney $U$-statistic ( $p$ value $\leq 0.10$ ). So, the three-factor CAPM overestimates the average market beta, perhaps because the FFC pricing factors provide appropriate corrections to market beta (see Ferguson and Shockley, 2003).

The coefficients $\lambda_{1, F X, i}$ and $\lambda_{3, F X, i}$ respectively capture the FX rate exposures for firm $i$ under the threeand six factor CAPMs (see Eqs. (1a) and (2a)). For U.S. firms as a whole, $R_{F X, t-1}$ has both positive and negative effects on excess returns. The average values for both $\lambda_{1, F X, i}$ and $\lambda_{3, F X, i}$ are larger in absolute value when negative, than when positive. The average value for $\lambda_{3, F X, i}$ is larger than the average value for $\lambda_{1, F X, i}$ when positive, whereas, the average value for $\lambda_{1, F X, i}$ is larger than the average value of $\lambda_{3, F X, i}$ when negative. The difference between the magnitude of the coefficients of similar sign is not significant $(U$ statistic; $p$-value $>0.10)$. A larger proportion of firms have negative $\lambda_{1, F X, i}$ and $\lambda_{3, F X, i}$ coefficients than positive coefficients. Thus, $60.86 \%$ of all firms have negative $\lambda_{1, F X, i}$ coefficients while $55.00 \%$ have negative $\lambda_{3, F X, i}$ coefficients. ${ }^{10}$

\section{[Table 1, about here]}

On a firm-by-firm basis, only $16.78 \%$ (204 out of 1,216) of all U.S. firms have significant $\lambda_{1, F X, i}$ coefficients (see Table 1, Panel A), whereas only $15.08 \%$ (184 out of 1,220) of all U.S. firms have significant $\lambda_{3, F X, i}$ coefficients. Choi and Prasad (1995) find comparable results for contemporaneous FX rate effects in monthly returns. Differences in the magnitude of $\lambda_{1, F X, i}$ and $\lambda_{3, F X, i}$ for coefficients of the same sign are

\footnotetext{
${ }^{10}$ The proportion of U.S. firms reported to have negative FX rate exposure varies in prior studies. Using a sample of 1,156 U.S. firms, Hutson and Stevenson (2010) find that 33.33\% of the firms exhibit negative contemporaneous FX rate effects. Faff and Marshall (2005) find that $60 \%$ of the 35 U.S. firms in their sample exhibit negative contemporaneous FX rate effects. Pantzalis et al. (2001) find that $71 \%$ of their 220 U.S. firms exhibit negative FX rate effects. Sensible comparisons are difficult to make due to differences in the methodology, sample size, and the period of study.
} 
not statistically significant. Also, $75.49 \%$ (154 out of 204) of the significant $\lambda_{1, F X, i}$ coefficients are negative, whereas $67.39 \%$ (124 out of 184) of the significant $\lambda_{3, F X, i}$ are negative. So, more U.S. firms have significant and negative FX rate effects than positive FX rate effects. This result reflects the pattern in our aggregate results.

Interest rate changes, $R_{f, t-1}$ also have both positive and negative effects on excess returns. Across the CAPMs, the $\gamma_{1, f, i}$ and $\gamma_{2, f, i}$ coefficients are very similar in magnitude ( $U$-statistic; $p$-value $>0.10$ ). So both CAPMs capture interest rate effects to a similar extent. The magnitude of the average $\gamma_{1, f, i}$ and $\gamma_{2, f, i}$ coefficients indicates that $R_{f, t-1}$ has a weaker effect on excess returns than $R_{F X, t-1} \cdot{ }^{11}$ Across both bivariate models, the majority of firms (between $56.15 \%$ and $60.94 \%$ ) are negatively impacted by interest changes.

Only $20.72 \%$ (252 out of 1,216) of the individual firms have significant $\gamma_{1, f, i}$ coefficients for $R_{f, t-1}$ (firmby-firm); 61.11\% (154 out of 252) of those coefficients are negative. Fewer firms (17.13\%) have significant $\gamma_{2, f, i}$ coefficients and here, $56.46 \%$ of these $\gamma_{2, f, i}$ coefficients are negative. The difference in the magnitude of $\gamma_{1, f, i}$ and $\gamma_{2, f, i}$ for coefficients of similar sign is not significant ( $U$-statistic; $p$-value $>0.10$ ). So both CAPMs perform equally well in capturing the magnitude of interest rate effects although there is variation in the number of firms with interest rate effects according to the coefficient sign. Even so, we do not claim the FFC pricing factors eliminate all potential mispricing (see Petkova, 2006) in the six-factor CAPM.

Almost $80 \%$ of the firms are positively exposed to $S M B_{t}$, whereas $76.74 \%$ are positively exposed to $M O M_{t}$. In contrast, $50.08 \%$ of the firms are positively exposed to $H M L_{t}$. So both $S M B_{t}$ and $M O M_{t}$ contain more economic information than $H M L_{t}$. On a firm-by-firm basis, $66.23 \%$ of the firms are significantly exposed to $S M B_{t}$, whereas $56.63 \%$ are significantly exposed to $H M L_{t}$. Only $17.54 \%$ are significantly exposed to $M O M_{t}$. So the FFC factors have varying effects on excess returns. The degree of the exposure to the FFC factors is economically important because we show later that as the number of firms with significant $M O M_{t}$ coefficients increases with the length of the return horizons, the number of firms with significant $S M B_{t}$ and $H M L_{t}$ decreases.

\footnotetext{
${ }^{11}$ Bodnar et al. (1998) show that $83 \%$ of U.S. firms use derivatives to manage FX rate risk, whereas $76 \%$ also use derivatives to manage interest rate risk; interest rate risk being the second most important risk managed by U.S. firms.
} 
Under the three-factor CAPM, more firms $(173$ out of 1,216) exhibit significant coefficients for the crisis variable, $D_{t}$, than under the six-factor CAPM $\left(152\right.$ out of 1,220). ${ }^{12}$ Also, the coefficients for $\lambda_{2, F X, i}$ and $\lambda_{4, F X, i}$ in the FX rate equations are typically positive and significant (see Eqs. (1b) and (2b)). About $83 \%$ of the $\lambda_{2, F X, i}$ and $\lambda_{4, F X, i}$ coefficients are significant in each case. So $R_{F X, t-1}$ has strong predictive effects on $R_{F X, t}$, in contrast to its effects on excess stock returns. About $44 \%$ of the conditional correlations $\left(\rho_{12}\right.$ and $\left.\rho_{34}\right)$ in each bivariate model are significant. This percentage is between 2.68 and 2.95 times larger than the percentage of firms with significant FX rate effects depending on the CAPM. We explore the connection between the conditional correlations and the presence of FX rate and interest rate effects in a later section.

\subsubsection{Coefficients of mean equations by firm size}

Generally, small firms tend to be more exposed to FX rate and interest rate effects than large firms (Block and Gallagher, 1986; Dominguez and Tsar, 2006). One reason is that large firms have a greater capacity to manage and hedge their exposures than small firms. However, a larger proportion of large U.S. firms use derivatives compared to the proportion of small firms that do (Bodnar et al., 1996). Tests for firm size are important, since firm size impacts on the ability of firms to use hedging techniques or create natural hedges. We test for the effects of firm size by categorizing all firms as either, large, medium, or small using their market capitalization value. ${ }^{13}$ We then match the exposure coefficients to the firms in each group.

Table 2 shows that the magnitude of the coefficients varies across both firm sizes and CAPMs. Irrespective of size, more firms carry negative $\lambda_{1, F X, i}$ and $\lambda_{3, F X, i}$ coefficients than positive $\lambda_{1, F X, i}$ and $\lambda_{3, F X, i}$ coefficients. As predicted, the average (for both positive and negative) coefficients for $\lambda_{1, F X, i}$ and $\lambda_{3, F X, i}$ are larger for small firms (see Panels A and B). The averages for $\lambda_{1, F X, i}$ and $\lambda_{3, F X, i}$ decrease monotonically, as firm size increases, except for large firms with positive effects under the three-factor CAPM. Hutson and Stevenson (2010) find comparable results.

[Table 2, about here]

\footnotetext{
12 The coefficients for relative risk aversion, $\delta_{1, i}$ and $\delta_{2, i}$ are larger under the six-factor CAPM, perhaps reflecting the ability of this model to capture better underlying economic conditions. Estimates for relative risk aversion vary substantially in empirical work (see, e.g., Brandt and Wang, 2003).

${ }^{13}$ To do this, we use the fiscal year-end price per share multiplied by the number of shares outstanding from the Compustat database, that is, item \#199 $\times$ item \#25. We then sort the firms according to the three groups. The use of the Compustat database results in a small loss in the number of firms (less than 6\%) relative to the total number of firms in our original sample. This is because the procedure entails matching the firms from the CRSP database with those from Compustat.
} 
Specifically, the average value of $\lambda_{1, F X, i}$ is $0.138 \%$ for small firms when positive, and $-0.207 \%$ when negative. Both averages are significantly different from zero ( $p$-value $<0.01)$. These coefficients are 1.28 (when positive) and 1.72 (when negative) times larger than those of large firms. The differences in the magnitude of the coefficients of small and large firms are statistically significant $(U$-statistic; $p$-value < 0.05). The positive and negative $\lambda_{1, F X, i}$ coefficients for small firms are also larger than the corresponding positive and negative $\lambda_{1, F X, i}$ coefficients for medium firms $(U$-statistic; $p$-value $<0.05)$. Medium firms also have larger negative $\lambda_{1, F X, i}$ coefficients than large firms with negative $\lambda_{1, F X, i}$ coefficients $(p$-value $<0.01)$. However, the $\lambda_{1, F X, i}$ coefficients for medium and large firms are similar in magnitude when positive ( $p$ value $>0.10$ ). On a firm-by-firm basis, more medium firms have significant FX rate coefficients, compared with small and large firms under the three-factor CAPM. This is unexpected but may reflect differences in the level of managerial risk aversion across firm sizes.

For the six-factor CAPM, the magnitude of the positive and negative $\lambda_{3, F X, i}$ coefficients for small firms is larger than those of both medium and large firms ( $U$-statistic; $p$-value $<0.05)$. More firms are affected by the negative effects of FX rate changes than by their positive effects. Medium-sized firms behave more like large firms under the six-factor CAPM; their $\lambda_{3, F X, i}$ coefficients are not significantly different irrespective of the coefficient sign $(U$-statistic; $p$-value $>0.10)$.

Across the CAPMs, the $\lambda_{1, F X, i}$ and $\lambda_{3, F X, i}$ coefficients are similar in size ( $U$-statistic; $p$-value $>0.10$ ), except for the positive and negative $\lambda_{1, F X, i}$ and $\lambda_{3, F X, i}$ coefficients associated with medium-sized firms ( $U$-statistic; $p$-value $<0.10)$. It is unclear why only medium-sized firms exhibit FX exposure of a different order of magnitude across the CAPMs. Even so, there is not much consistency in the magnitude of the average coefficients based on common coefficient sign across the CAPMs.

Differences in the degree of FX rate effects by firm size can reflect two factors. First, large and mediumsized firms (to some extent) are able to allocate more resources to manage risk than small firms can. In addition, large and medium-sized firms are also more geographically spread out than small firms are (Marston, 2001). Both considerations give large and medium-sized firms greater opportunities to offset and manage their exposures relative to small firms (Pantzalis et al., 2001). Second, since large firms hedge more than small firms (Bodnar et al., 1996), FX rate changes will have a smaller impact on the excess returns of 
large firms. If large and medium-sized firms have greater competitive strength in export and import markets and they can enforce stronger pricing-to-market strategies, they will also exhibit less FX rate effects.

For the three-factor CAPM, 53.40\% of medium firms have negative $\gamma_{1, f, i}$ coefficients compared with around $64.92 \%$ for small and large firms. Similarly, for the six-factor CAPM, $49.48 \%$ of medium firms have negative $\gamma_{2, f, i}$ coefficients compared with $59.21 \%$ for small and large firms (see Table 2). Medium firms are less geographically spread out than large firms and perhaps are more active in managing the negative effects of interest rate changes. Across the CAPMs and firm sizes, the magnitude of the $\gamma_{1, f, i}$ and $\gamma_{2, f, i}$ coefficients for similar coefficient sign is not typically significant. The exceptions are negative the $\gamma_{1, f, i}$ and $\gamma_{2, f, i}$ coefficients for medium-sized firms (as a pair) and the positive $\gamma_{1, f, i}$ and $\gamma_{2, f, i}$ coefficients (as a pair) for large firms ( $U$-statistic; $p$-value $<0.05)$. So there is some variation in the results for both medium and large firms for interest rate changes.

The financial crisis had a larger negative effect than a positive effect on excess returns. The average positive and negative coefficients for $\xi_{1}$ and $\xi_{2}$ decrease monotonically, as firm size increases. The differences are significant for similar coefficient sign ( $U$-statistic; $p$-value $<0.05)$. As such, large firms were more resilient against the effects of the financial crisis. Conversely, small firms were more exposed to the effects of the financial crisis than other firm sizes.

The average $\rho_{12}$ and $\rho_{34}$ conditional correlations are non-zero and vary across bivariate models. Since $\rho_{12}$ and $\rho_{34}$ are non-zero, their magnitude can be regarded as an additional source of residual exposure. ${ }^{14}$ The $\rho_{12}$ and $\rho_{34}$ conditional correlations are larger for large firms and generally smaller for small firms ( $p$-value $<0.10)$. Since $\lambda_{1, F X, i}$ and $\lambda_{3, F X, i}$ are smaller for large firms and their $\rho_{12}$ and $\rho_{34}$ conditional correlations are larger, there seems to be a negative relation that may be linked with firm size. So, lower FX rate effects may be associated with the volatility of excess returns and FX rate changes when the volatility of these variables is highly correlated. We explore this issue below.

\subsection{Coefficients of mean equations and short-horizon returns}

Long-horizon studies often focus on FX rate effects as opposed to interest rate and other effects. We consider the patterns in our results for all the variables in our bivariate equations. The results for long-

\footnotetext{
${ }^{14}$ We thank an anonymous reviewer for pointing this out to us.
} 
horizon studies do not appear to be due to data mining and/or artifacts of the sample, since similar results have been reported for other countries (see, e.g., Dominguez and Tesar, 2006). These studies consistently use overlapping monthly price changes, except for Muller and Verschoor (2008) who use weekly overlapping returns. The use of overlapping price changes can induce a moving average error that is correlated with the return horizon. Since the price changes are likely to be stationary, rolling sums of the returns can give rise to the well-known spurious correlation problem (see Ferson et al., 2003) and persistence in the returns (see Boudoukh et al., 2006). Long-horizon studies adjust for the residual autocorrelation and heteroscedasticity that can occur in overlapping returns (Chow et al., 1997a, 1997b; Bodnar and Wong, 2003). However, measures like the Newey-West, only adjust the standard errors while leaving the estimated coefficients unchanged. So it is not clear whether these adjustments are adequate or appropriate. We use non-overlapping price changes to avoid some of these problems. We report the results only for the coefficients that are statistically significant firm-by-firm.

\subsubsection{Mean equations of CAPMs under constant conditional correlation}

Panels A and B of Figure 1 show the bar charts representing the number of firms (frequency) with statistically significant coefficients at $d$-periods. From $d=1$ to $d=40$, the number of firms with significant $\alpha_{1, i}$ coefficients increases by $103.78 \%$ (185 to 377 firms) for the three-factor CAPM, whereas, the increase is $126.89 \%$ (212 to 481 firms) for $\alpha_{3, i}$ (under the six-factor CAPM). ${ }^{15}$ The percentage increases are not linearly related with the $d$-periods. For example, at $d=1$ and 14, the number of firms with significant $\alpha_{3, i}$ coefficients is lowest. Across both CAPMs, the increase in the number of significant coefficients is often due to a larger proportion of the coefficients becoming positive at certain horizons, as the return horizon increases. Similar to the long-horizon studies (see, e.g., Dominguez and Tesar, 2006), the number of firms with significant $\lambda_{1, F X, i}$ and $\lambda_{3, F X, i}$ coefficients increases with the length of the return horizon. The increase for $\lambda_{1, F X, i}$ is $61.27 \%$ by $d=40$ (i.e., 204 to 329 firms) relative to $d=1$, whereas, the increase for $\lambda_{3, F X, i}$ is

\footnotetext{
15 Theoretically, average $\alpha_{1, i}$ and $\alpha_{3, i}$ should be zero at all return horizons as this would mean that the explanatory variables span the minimum-variance tangency portfolio (see Fama and French, 2012). The average $\alpha_{1, i}$ and $\alpha_{3, i}$ are not zero even when aggregation includes all firms in the sample whether or not $\alpha_{1, i}$ and $\alpha_{3, i}$ are individually significant for each CAPM. Since average $\alpha_{1, i}$ and $\alpha_{3, i}$ are not zero for aggregation based on individually significant cases, this indicates that as a portfolio, the stocks are not fairly priced, as the equilibrium return is different from what would have been predicted by CAPM.
} 
$126.63 \%$ (184 to 417 ) by $d=40$ relative to $d=1$. Thus, although starting from a lower base, the six-factor CAPM outperforms the three-factor CAPM. Across all return horizons the number of positive and negative $\lambda_{1, F X, i}$ coefficients is not evenly spread. Overall, the six-factor CAPM generates $3 \%$ more firms with negative $\lambda_{3, F X, i}$ coefficients. So under the six-factor CAPM, for example, negative $\lambda_{3, F X, i}$ coefficients dominate positive $\lambda_{3, F X, i}$ coefficients at $d=1,11$ to 20 , and 40 . Our finding also suggests that there is a tendency for the number of positive and negative coefficients to even out in the long run. While we do not show this result, the averages for $\lambda_{1, F X, i}$ and $\lambda_{3, F X, i}$ also increase with the return horizons (see also, Chow et al., 1997a). ${ }^{16}$ Variation in exposure effects can reflect differences in the maturity cycles of the hedging instruments firms use.

\section{[Figure 1, about here]}

The percentage increase in FX rate effects from $d=1$ to $d=2$ is $14.71 \%$ (29.35\%) for the three-factor (sixfactor) CAPM. No horizon-on-horizon increases exceed this value except at $d=17$ and $d=30$ for the threefactor CAPM, and $d=20$ for the six-factor CAPM. So, one of the largest percentage increases in FX rate effects is at the earliest return horizon. This finding gives some support to our view that firms may be less effective in hedging short-horizon returns (see also Footnote 5). The comparisons are however not entirely fair since the length of the $d$-periods is not constant. Nevertheless, it seems reasonable to suggest that FX rate effects increase at a decreasing rate over time. So the percentage increase as early as, by $d=2$ is followed by smaller percentage increases in most cases, as the return horizons increase. This finding casts some doubts on the view that the weak support for FX rate effects in one-period returns, is due to the good hedging skills of firms. Using our results, one can also reasonably argue that U.S. firms are somewhat better at hedging their exposures at more distant horizons, simply because the increase in the positive and negative exposures is more or less at a decreasing rate after $d=2$. Furthermore, if U.S. firms hedge cash flow exposures over a two-to-four-quarter horizon (see Dolde, 1993), well outside the horizons we analyze, it is possible for them to be less effective at hedging at very short return horizons. Our findings can mean at least two things: i) there is an underlying residual exposure that cannot be captured by the risk management efforts of firms; ii) risk management has a decreasing effect on exposure over-time and this decreasing effect leads to the accumulation of more exposure that is captured at longer return horizons.

For the three- and six-factor CAPMs, the total increase in the number of significant $\lambda_{1, F X, i}$ and $\lambda_{3, F X, i}$ coefficients (i.e., from $d=1$ to $d=40$ ) is respectively $27.06 \%$ (329 out of 1,216 ) and $34.18 \%$ (417 out of

\footnotetext{
${ }^{16}$ For the three-factor CAPM, the averages of the positive (negative) FX rate coefficients are $0.32 \%(-0.35 \%)$ for day 1 , and $1.50 \%$ ($1.97 \%)$ day 40 . The corresponding percentages for the six-factor CAPM are $0.05 \%(-0.10 \%)$ at day 1 and $0.26 \%(0.31 \%)$ at day 40 . The differences across returns horizons for each CAPM are statistically significant.
} 
1,220) of the full sample. These percentages are only slightly better than those of prior studies if we interpolate eight weeks (equivalent to 40 days) from the intervals used by Bodnar and Wong (2003) and Muller and Verschoor (2006b). So the relation between the number of firms with FX rate effects and the length of the return horizons seems to hold approximately.

Figure 1 also shows that the number of firms with significant interest rate effects increases with the return horizons. From $d=1$ to $d=40$, we find a $73.81 \%$ (252 to 438 ) increase in the number of firms with significant $\gamma_{1, f, i}$ coefficients. For $\gamma_{2, f, i}$, the increase is $145.93 \%$ (209 to 514). Similar to FX rate effects, the largest percentage increase in the number of firms with significant $\gamma_{1, f, i}$ and $\gamma_{2, f, i}$ coefficients is at $d=2$ relative to $d=1$. The increase is $80.16 \%$ for $\gamma_{1, f, i}$ and $112.44 \%$ for $\gamma_{2, f, i}$ (comparing $d=2$ and $d=1$ ). By $d=40,36.02 \%$ of the full sample of firms $(438$ out of 1,216$)$ have significant $\gamma_{1, f, i}$ coefficients, whereas, 42.13\% (514 out of 1,220) of the full sample of firms have significant $\gamma_{2, f, i}$ coefficients. So, like FX rate effects, the number of firms with interest rate effects also increases with the length of the return horizons.

Overall, these findings reaffirm our view that the good hedging skills of managers cannot fully explain the weak support for FX rate and interest rate effects in one-period returns. If hedging short maturities is too costly given the associated risk (Fehle and Tsyplakov, 2005), firms are less likely to hedge short-horizon exposures than long horizon exposures. This is because $d$-periods of less than 40 days or even two days seem too short for risk management to be effective and our $d$-periods are quite out of line with the typical hedging horizons used in practice.

Interestingly, the number of firms with significant risk tolerance coefficients, i.e., $\delta_{1, i}$ and $\delta_{2, i}$ decline over time for both CAPMs. As expected, investors' perception of risk, measured by the magnitude of $\delta_{1, i}$ and $\delta_{2, i}$, is lower at longer return horizons, perhaps, because excess returns are more predictable and less risky at longer horizons. While investors may believe that corporate hedging reduces exposure, they may not adequately re-evaluate their risk profiles in respect of the accumulation of exposures over time. Otherwise, our risk tolerance coefficients would increase both in magnitude and the frequency of the significant coefficients, as the return horizons increase. Across the CAPMs, the coefficients of the explanatory variables of similar sign are often significantly different in pair-wise comparisons - especially for $d=14$ days or less $(U$-statistic, $p$-value $<0.10)$.

For the FFC pricing factors, the number of firms with significant $S M B_{t}$ and $H M L_{t}$ coefficients steadily decreases as the return horizons increase. Conversely, the number of firms with significant $M O M_{t}$ coefficients increases with the return horizon. From $d=1$ to $d=40$, the decrease in the number of 
significant $S M B_{t}$ and $H M L_{t}$ coefficients is about $30 \%$, whereas the increase is $92.99 \%$ for $M O M_{t}$. So while fewer firms are exposed to $S M B_{t}$ and $H M L_{t}$ as the return horizons increase, more firms are exposed to $M O M_{t}$ in line with the results for FX rate and interest rate effects. Similar to our exposure results, the largest percentage increase in the number of significant $S M B_{t}$ and $H M L_{t}$ coefficients is at $d=2$. Thereafter, the number of significant $S M B_{t}$ and $H M L_{t}$ coefficients decreases as the return horizons increase. The largest single percentage increase in the number of firms with significant $M O M_{t}$ coefficients is at $d=40$ (relative to $d=1$ ). If $S M B_{t}$ and $H M L_{t}$ capture the effects of macroeconomic surprises (Simpson and Ramchander, 2008), these surprises become less important at longer horizons. The coefficient sign for $S M B_{t}$ and $M O M_{t}$ is typically positive whereas, the coefficient sign for $M O M_{t}$ is more evenly distributed. Even so, these variables impact on excess returns are varying rates as the length of the return horizons increases.

\subsubsection{Mean equations of FX rate equations under constant conditional correlation}

Panel C of Table 1 shows that about $83 \%$ of the total $\lambda_{2, F X, i}$ and $\lambda_{4, F X, i}$ coefficients in the FX rate equations are significant for day 1, in each case (see Eqs. (1b) and (2b)). At $d=2$, no more than $8 \%$ of the $\lambda_{2, F X, i}$ and $\lambda_{4, F X, i}$ coefficients (in each case) are significant. The percentages for the number of significant $\lambda_{2, F X, i}$ and $\lambda_{4, F X, i}$ coefficients relative to the total sample, diminish rapidly. So for $\lambda_{2, F X, i}$, less than $1 \%$ of the total $\lambda_{2, F X, i}$ coefficients are significant at $d=8$, although the percentage increases, but by no more than $36.10 \%$ at $d=30 .{ }^{17}$ There is no evidence of an increase in the number of significant $\lambda_{2, F X, i}$ and $\lambda_{4, F X, i}$ coefficients from $d=1$ to $d=2$, in contrast to the CAPM results. So changes in FX rates, $R_{F X, t-1}$ exhibit quick price reversals. This result is unexpected, given that FX rate effects increases with the return horizon in the CAPMs.

\subsubsection{Constant conditional correlation coefficients}

Baillie and Bollerslev (1989) find that the conditional volatility in high frequency returns is higher than that in low frequency returns. If the conditional volatility of excess returns and FX rate changes responds to the content of information flows, the conditional correlations obtained from their volatility estimates will be higher at the shortest return horizon, because uncertainty in information flows will be highest at

\footnotetext{
${ }^{17}$ Froot and Ramadorai (2005) also find short-term momentum (under-reaction) and long-term reversals in bilateral FX rate price changes for up to 30 trading days.
} 
these intervals (see, Aslanidis et al., 2010). Conversely, the size of the conditional correlations and the number of significant conditional correlations will be lower at longer return horizons because the news impacts will be less correlated with returns. Overall, we expect the number of firms with significant conditional correlations to be negatively correlated with the number of firms with significant exposure effects. As such, both $h_{12, t}=\rho_{12}\left(h_{1, i, t}, h_{2, i, t}\right)^{1 / 2}$ and $h_{34, t}=\rho_{34}\left(h_{3, i, t}, h_{4, i, t}\right)^{1 / 2}$ (see Eqs. (5a) and (5b)) will have stronger impacts at the shortest return horizons when exposure effects are weakest.

Panel D of Figure 1 shows our results. As predicted, the number of firms with significant $\rho_{12}$ and $\rho_{34}$ conditional correlations declines as the return horizons increase. As before, roughly $44 \%$ of the $\rho_{12}$ and $\rho_{34}$ conditional correlations are significant at $d=1$ (in each case), relative to the full sample. The number of significant $\rho_{12}$ and $\rho_{34}$ conditional correlations falls by between $22 \%$ and $30 \%$ from $d=1$ to $d=2$. This is precisely the same interval at which we find a very large percentage increase in the number of firms with significant exposure effects. By $d=14,85.90 \%$ less $\rho_{12}$ conditional correlations are significant relative to $d$ $=1$. For $\rho_{34}$, the decline is $91.14 \%$ (for $d=14$ compared with $d=1$ ). Thereafter, there is a gradual increase in the number of significant $\rho_{12}$ and $\rho_{34}$ conditional correlations, giving rise to a $U$-shape pattern. But this $U$-shape is largely a distortion in our data interval. By $d=40$, the number of significant $\rho_{12}$ and $\rho_{34}$ conditional correlations is lower by between $61.54 \%$ and $70.33 \%$ relative to $d=1$.

We use the Spearman rank correlation, $r_{s}$ to test for an association between the number of firms with significant conditional correlations and the number of firms with significant exposure effects. This test does not imply causation. For the three-factor CAPM, $r_{s}$ is positive and significant for the number of negative $\rho_{12}$ conditional correlations and the number of negative $\lambda_{1, F X, i}$ coefficients $\left(r_{s}=0.707 ; p\right.$-value $=$ 0.022). We find a similar result for the total number of significant conditional correlations, $\rho_{12}$ and the number of negative $\lambda_{1, F X, i}$ coefficients $\left(r_{s}=0.665 ; p\right.$-value $\left.=0.036\right)$. The $r_{s}$ statistic is also positive for the number of $\rho_{12}$ coefficients that is negative compared with number of firms with positive $\gamma_{1, f, i}$ coefficients $\left(r_{s}=0.590 ; p\right.$-value $\left.=0.073\right)$. We find a similar result for the number of negative $\rho_{12}$ coefficients and the total number of firms with positive and negative $\gamma_{1, f, i}\left(r_{s}=0.614 ; p\right.$-value $\left.=0.059\right)$ and corresponding results for $\rho_{34}$ and $\gamma_{2, f, i}$ are not significant. So these findings are contrary to expectation. We believe that this contrary result is partly due to the distortion in the horizon-on-horizon 
interval beyond $d=20$, given also the pattern in the bar charts (see Figure 1, Panel C). Of course, the small tendency for more firms to be exposed to negative than positive exposure effects as the return horizons increase can also impacts on the sign of the $r_{s}$ statistic.

However, the $r_{s}$ statistic is positive and often significant ( $p$-value $\left.=0.080\right)$ when the total number of (positive and negative) $\rho_{12}$ correlations is paired with the total number of positive and negative coefficients for long-term volatility, past news and conditional volatility (in each case). We find similar results for $\rho_{34}$. So, the decline in the number of firms with significant $\rho_{12}$ and $\rho_{34}$ is also linked with a decline in the number of significant coefficients in our variance equations. This result is not surprising since the conditional variance (volatility) used to construct the conditional correlations are the same measures used to generate the coefficients for the variance equations.

Our samples of significant cases are small and the intervals of the return horizons beyond $d=20$ are larger than at earlier horizons. This hides some of the effects we wish to observe. As such, we use a bootstrap method (see Efron, 1979) of sampling with replacement to validate the above results. So, we draw

random resamples of $X^{*}=\left(x_{1}^{*}, x_{2}^{*}, \ldots, x_{n}^{*}\right)$ from $X=\left(x_{1}, x_{2}, \ldots, x_{n}\right) 1,000$ times for each return horizon and bivariate model, using coefficients of similar sign.

In general, the bootstrap results show that a similar percentage of firms exhibits significant exposure effects across all coefficients, compared with our raw results. This means that our inferences based on the number of firms with significant coefficients seem reliable. There are some variations in the magnitude of the average coefficients of our bootstrap results relative to our raw values. However, in many cases, these differences are not statistically significant.

\section{3. $\quad$ Smooth transition estimates}

To run our STCC-GJR-GARCH-M model, we first need to test for a structural break in the excess return series. Structural breaks are identified using the $\mathrm{LM}_{\mathrm{cc}}$ statistic. We present the results below for both bivariate models.

\subsubsection{Coefficients of mean equations under smooth transition conditional correlation}

Figure 2 shows the results for the mean equations under the STCC-GJR-GARCH-M. Panels A and B show that at $d=1$, the percentages of significant $\lambda_{1, F X, i}$ and $\lambda_{3, F X, i}$ coefficients are quite small. The significant cases represent less than $2 \%$ of the full sample of firms, when also their excess returns contain a structural break. The number of firms with structural breaks in their excess returns increases with the return horizons such that between $9 \%$ and $13 \%$ of the firms contain structural breaks at $d=40$. These percentages are still 
small. Even so, by $d=40,710 \%$ (400\%) more firms have significant $\lambda_{1, F X, i}$ and $\lambda_{3, F X, i}$ coefficients relative to $d=1$.

As before, the increase in the return horizons from $d=1$ to $d=2$ contains the largest percentage increase in the number of firms with significant FX rate effects, unless the percentage change is preceded by a substantial decline. Unlike the constant conditional correlations case, the STCC-GJR-GARCH-M generates more firms with positive than negative coefficients for $\lambda_{1, F X, i}$, whereas, the number of positive and negative $\lambda_{3, F X, i}$ coefficients are more evenly spread. However, at $d=11$ for $\lambda_{1, F X, i}$ and $d=11,17,20$, and 40 for $\lambda_{3, F X, i}$, more coefficients are negative than positive - a finding partly in line with the constant conditional correlation case.

The number of firms with significant interest rate effects also increases with the return horizons. By $d=40$, $395.35 \%$ more firms have significant $\gamma_{1, f, i}$ coefficients relative to $d=1$. For the $\gamma_{2, f, i}$, coefficients, the percentage is $176.60 \%$. As before, the largest percentage increase in interest rate effects is at $d=2$ relative to $d=1$.

\section{[Figure 2, about here]}

In contrast to our earlier results, there is more variability in the percentage of firms with significant $S M B_{t}$, $H M L_{t}$ and $M O M_{t}$ coefficients. The rate of decline for the number of firms with significant $S M B_{t}$ coefficients is less steady compared with the constant conditional correlation case. There are more negative $H M L_{t}$ coefficients than positive coefficients. The number of significant coefficients for $M O M_{t}$ still increases at longer return horizons but only after a substantial decline at around $d=8$. As before, the largest number of significant $\lambda_{2, F X, i}$ and $\lambda_{4, F X, i}$ coefficients for the FX rate equations is at $d=1$ (see Panel C).

\subsubsection{Structural breaks and $F X$ rate effects}

We also examine whether firms with structural breaks in their excess returns are more likely to exhibit significant FX rate effects under the STCC-GJR-GARCH-M. Table 3 shows that for day 1, firms with significant structural breaks are less likely to exhibit significant FX rate effects by a ratio of 7 to 1 . In this case, around $15 \%$ of the firms with significant structural breaks also exhibit significant FX rate effects for both CAPMs. This represents less than $2 \%$ of the full sample of firms under each CAPM. The number of firms with both significant structural breaks and FX rate effects increases with the return horizons. At $d=$ 40 , the ratio of the number of firms with structural breaks compared with FX rate effects is reduced substantially to 3.494 for the three-factor CAPM and 2.655 for the six-factor CAPM. So, more structural 
breaks in the excess returns do not lead to proportionately more firms with FX rate effects. The $r_{s}$ statistic is positive ( $p$-value $<0.02$ ), confirming a positive relation between the number of firms with structural breaks and those with FX rate effects under each CAPM.

\section{[Table 3, about here]}

\subsubsection{Significant coefficients of the smooth transition function}

Figure 3 plots the number of significant coefficients for the transition function at each return horizon. The patterns in the conditional correlations are not as pronounced as those of the constant conditional correlation. The number of significant conditional correlations increases with the return horizons especially for the bivariate case containing the three-factor CAPM when the conditional correlations increase in value. This is less so for the six-factor CAPM. Except for the smoothness coefficient, negative coefficients dominate as the return horizons increase. Overall, these findings provide less support for the pattern we predicted for the conditional correlations. Of course, the small sample size can impact on our results.

\section{[Figure 3, about here]}

Table 5 shows the coefficients for the STCC transition function according to the particular regime. A positive conditional correlation means that $\rho_{12 a}>\rho_{12 b}$, whereas a negative conditional correlation means that $\rho_{12 b}<\rho_{12 a}$ (see Eqs. (6) and (8)). The average conditional correlations are both negative and positive and their magnitude increases with the return horizons. For the three-factor CAPM, the minimum average conditional correlation in regime $\rho_{12 a}$ for $\rho_{12 a}>0$ is -0.088 (at $d=1$ ). The maximum average conditional correlation in regime $\rho_{12 a}$ for $\rho_{12 a}>0$ is -0.281 (at $d=30$ ). The parameter $c_{1}$ is within the range of -0.043 (at $d=1$ ) to -0.427 (at $d=40$ ). When $R_{F X, t-1}$ crosses the threshold point at $c_{1}$, the correlation moves to regime $\rho_{12 b}$. In regime $\rho_{12 b}$, the minimum conditional correlation for $\rho_{12 b}<0$ is $-0.066($ at $d=1)$, whereas the maximum conditional correlation in regime $\rho_{12 b}$ for $\rho_{12 b}<0$ is -0.255 (at $d=40$ ). For both regimes, the range between the minimum and maximum points is quite wide. This means that the crossover into a given regime is likely to be very abrupt. The minimum conditional correlations for $\rho_{12 a}$ and $\rho_{12 b}$ are at the

shorter return horizons, whereas the maximum conditional correlations are at long return horizons. This suggests that small FX rate effects accumulate over time, leading to more FX rate effects, in line with our earlier results (see Section 5.2.1). A similar pattern is observed for the six-factor CAPM, except that the conditional correlations are larger and their changes based on $c_{2}$ are more abrupt. 


\section{[Table 5, about here]}

\subsection{Conditional variances at long return horizons}

We now consider the coefficients for the conditional variance of both bivariate equations. Both conditional volatility and asymmetry directly affect stock returns (Nelson, 1990). Asymmetric volatility - the leverage effect - follows from the disproportionate effects that bad news has on the volatility of returns compared with good news. If volatility arises in response to the information contained in news items (see Ross, 1989), conditional volatility and asymmetry will have greater impacts both in their magnitude and frequency at short return horizons, thus making average stock returns more risky at these horizons. In this case, FX rate and interest rate effects might be reduced if the higher conditional volatility and asymmetry at short return horizons cause excess returns to be more negatively correlated with our exposure estimates. Estimates of conditional volatility are also important for investors when they construct dynamic hedging strategies and estimate value at risk (VaR). We focus on the cases where the coefficients in the variance equations are significant as this enables us to evaluate their relative impacts across the return horizons.

\subsubsection{Conditional variance under the constant conditional correlation}

Figure 4 shows the bar charts under the constant correlation assumption. As predicted, for most variables, the number of significant coefficients decreases as the return horizon increases (see Eqs. (3a) to (4b)). For both CAPMs, the coefficients for past news $\left(\phi_{1, i}\right.$ and $\left.\phi_{3, i}\right)$ exhibit a weak $U$-shape but this shape is distorted as before. The number of significant conditional volatility coefficients, i.e., $\varphi_{1, i}$ and $\varphi_{3, i}$ declines very slowly - more slowly than long-term conditional variance i.e., $\mu_{1, i}$ and $\mu_{3, i}$. The direction is in line with our prediction but the decline is a lot less rapid than we expected. The total number of significant (positive and negative) coefficients for conditional asymmetry, i.e., $\vartheta_{1, i}$ and $\vartheta_{3, i}$ increases at $d=2$ relative to $d=1$, but declines gradually at subsequent return horizons until $d=20$. The number of firms with positive conditional asymmetry takes on a similar pattern. The pattern for (reverse) conditional asymmetry $\left(\vartheta_{1, i}\right.$ and $\left.\vartheta_{3, i}\right)$ is less predictable and the number of firms with reverse conditional asymmetry increases substantially after $d=20$. The presence of reverse conditional asymmetry is inconsistent with the theory but may reflect the stock market's view about future news impacts.

\section{[Figure 4, about here]}


For the FX rate equations, the decline in the number of significant coefficients is very abrupt, particularly for past news $\left(\phi_{2, i}\right.$ and $\left.\phi_{4, i}\right)$ and conditional asymmetry $\left(\vartheta_{2, i}\right.$ and $\left.\vartheta_{4, i}\right)$. Negative coefficients for past asymmetry dominate, in most cases. The peaks and troughs in the frequency of significant cases are widespread and widely dispersed. The number of cases with significant conditional volatility ( $\varphi_{2, i}$ and $\left.\varphi_{4, i}\right)$ also declines at a relatively steady pace until $d=17$, after which the decline is abrupt.

We also apply the $r_{s}$ statistic to test the relation between the coefficients of the variance equations and our exposure effects. This test enables us to assess whether our measures of conditional volatility are associated with the number of firm with exposure effects. The theory would suggest that the volatility of excess returns and FX rate changes would impact on exposure effects at a decreasing rate as the return horizons increase.

In general, we find very weak support for our prediction in respect of the parameters of the three-factor CAPM. Specifically, the $r_{s}$ statistic is positive and significant for the number of firms with positive past news coefficients $\phi_{1, i}$ compared with the number of firms with negative $\lambda_{1, F X, i}$ coefficients $\left(r_{s}=0.632 ; p\right.$ value $=0.050$ ), contrary to our prediction. The results are somewhat better for the six-factor CAPM. Here, the $r_{s}$ statistic is negative and significant $\left(r_{s}=-0.839 ; p\right.$-value $\left.=0.002\right)$ for the relation between the number of firms with (positive) conditional volatility, $\varphi_{3, i}$ and the number of firms with positive $\lambda_{3, F X, i}$ coefficients. So, as the number of firms with significant $\varphi_{3, i}$ coefficients decreases, the number of firms with positive and significant $\lambda_{3, F X, i}$ coefficients increases. Similarly, the $r_{s}$ statistics is negative for the number of firms with (positive) conditional volatility, $\varphi_{3, i}$ and the number of firms with negative $\lambda_{3, F X, i}$ coefficients $\left(r_{s}=-\right.$ 0.927; $p$-value $=0.000)$. Given these results, the $r_{s}$ statistic is also negative for the number of firms with (positive) conditional volatility, $\varphi_{3, i}$ and the total number of firms with positive and negative $\lambda_{3, F X, i}$ coefficients $\left(r_{s}=-0.964 ; p\right.$-value $\left.=0.000\right)$.

For the three-factor CAPM, the $r_{s}$ statistic is not significant for the number of firms with significant coefficients in the variance equations and the number of firms with significant interest rate coefficients, $\gamma_{1, f, i}$. This result is in line with those of the three-factor CAPM for FX rate effects. For the six-factor CAPM, the $r_{s}$ statistic is negative for the number of firms with significant (positive) conditional volatility, $\varphi_{3, i}$ and the number of firms with positive $\gamma_{2, f, i}$ coefficients $\left(r_{s}=-0.614 ; p\right.$-value $\left.=0.059\right)$. 
Similarly, the $r_{s}$ statistic is negative for the number of firms with significant $\varphi_{3, i}$ coefficients and the number of firms with negative $\gamma_{2, f, i}\left(r_{s}=-0.588\right.$; $p$-value $\left.=0.074\right)$. Using the total number of firms with significant $\gamma_{2, f, i}$ coefficients and the number of firms with significant $\varphi_{3, i}$ also confirms the above result ( $r_{s}=-0.636 ; p$-value $\left.=0.048\right)$. Overall, our view that the number of firms with significant exposure coefficients is associated with measures of volatility is mainly supported by six-factor CAPM. Since we find that the number of firms with significant conditional volatility is strongest when the impact of information flows is likely to be strongest, the volatility in excess return can cause firms to alter their exposures in anticipation of the content of information flows.

\subsubsection{Conditional variance under the smooth transition model}

Under the STCC-GARCH-M, the bar charts (not shown) for the number of firms with significant coefficients in the variance equations display a similar pattern compared with the constant conditional correlation case. Compared with the constant conditional correlation case, there is more variation in the patterns of the variance equations for both CAPMs and the FX rate equations. The pattern is less predictable for the six-factor CAPM. However, most firms are dominated by negative asymmetry at longer return horizons. The patterns for variance equations of the FX rate equations are also less predictable.

\section{Conclusion and implications}

We estimate two bivariate models to test for a link between the volatility of both excess returns and FX rate changes with our exposure measures. We use short-horizon returns in contrast to prior work. For the threefactor CAPM, $61.27 \%$ (73.81\%) more U.S. firms exhibit significant FX rate (interest rate) effects by day 40, compared to day 1, under the constant conditional correlation assumption. Day 2 relative to day 1 exhibits more or less the largest increase in the number of firms with exposure effects. Further increases in exposure effects tend to be at a declining rate-a finding also supported by the STCC results. Our six-factor CAPM, which incorporates $S M B_{t}, H M L_{t}$, and $M O M_{t}$ generates weaker results for exposure effects at day 1. However, the six-factor CAPM outperforms the three-factor CAPM by day 40, by generating more firms with significant exposure effects. The six-factor CAPM also provides more consistent support for the relation between the number of firms with significant exposure effects and the number of firms with volatility coefficients that are significant. The bivariate STCC model generates the weakest results but some of the patterns in the results are in line with the constant conditional correlation case. The $r_{s}$ statistic does not provide statistical support for the negative relation we predicted between the number of significant conditional correlations and the number of firms with exposure effects although the relation appears to be 
negative when we look at the bar charts. The results based on the $r_{s}$ statistic are unexpected but may reflect the larger interval of our return horizons for more distant returns. Our inferences based on the $r_{s}$ statistic should not be taken to imply causation.

Our results are based on lagged FX rate and interest rate changes. While our results can be considered to be model specific, our approach is in line with the view that exposure effects may be observed with a lag (see Bartov and Bodnar, 1994), possibly by investors. We acknowledge that the lag in our exposure measures may not encompass the exact time period firms use to hedge their exposures. Despite this limitation, the exposure effects we observe may still be sufficiently representative to provide insights in the exposure problem. With this in mind, we can identify the following main contributions to prior work: i) Since we find that the frequency of our exposure effects increases with the length of our short horizon returns, this result appears to go against the argument that the weak support for exposure effects is due to the effectiveness of hedging by U.S. firms at short return horizons. ii) The negative relation between the number of firms with exposure effects and the frequency of significant conditional volatility may reflect the impacts of information flows which will be strongest at the shortest return horizons. Indeed, our findings seem in line with the predictions associated with uncertainty in information flows. Here, volatility is highest when markets are turbulent and lowest when they are calm (see Aslanidis et al., 2010). Since we find that conditional volatility is highest at the shortest return horizons - exactly when both the magnitude of the exposures and the smallest number of firms has exposure effects - the decrease in the frequency of significant volatility effects seems negatively associated with the frequency of exposure effects. Alternatively, prior work on volatility linkages and contagion effects (see Fleming et al., 1998) suggests that investors rebalance their portfolios possibly at short return horizons, in response to macro-economic shocks. This can give rise to the results we have found. So, exposure effects will be lower at short return horizons when conditional volatility is higher. However, empirical work does not provide much guidance on the twin activities of portfolio rebalancing and the corporate hedging activities of firms. 


\section{References}

Amihud, Y., 1994. Exchange rates and the valuation of equity shares. In Exchange Rates and Corporate Performance, Y. Amihud and R. Levich (eds.), pp. 49-59 (Irwin Professional Publishing, New York).

Aslanidis , N., Osborn, D.R. and Sensier, M., 2010. Explaining co-movements in US and UK stock prices: the role of international information. International Journal of Finance and Economics 15, 366-380.

Baillie, R., Bollerslev, T., 1989. The message in daily exchange rates: a conditional-variance tale. Journal of Business and Economic Statistics 7, 297-305

Banz, R., 1981. The relationship between return and market value of common stocks. Journal of Financial Economics 9, 3-18.

Bartov, E., Bodnar. G., 1994. Firm valuation, earnings expectations, and the exchange-rate exposure effect. Journal of Finance 44, 1755-1785.

Bartov, E., Bodnar, G., Kaul, A., 1996. Exchange rate variability and the riskiness of U.S. multinational firms: evidence from the breakdown of the Bretton Woods system. Journal of Financial Economics 42, $105-132$.

Bartram, S., 2002. The interest rate exposure of non-financial corporations. European Finance Review 6, 101125.

Bartram

, S., 2007. Corporate cash flow and stock price exposures to foreign exchange rate risk. Journal of Corporate Finance 13, 981-994.

Block, S., Gallagher, T., 1986. The use of interest rate futures and options by corporate financial managers. Financial Management 15, 73-78.

Bodnar, G., Hayt, G., Marston, R., 1996. 1995 Wharton survey of derivatives usage by US non-financial firms. Financial Management 25, 113-133.

Bodnar, G., Hayt, G., Marston, R., 1998. 1998 Wharton survey of financial risk management by US nonfinancial firms. Financial Management 27, 70-91.

Bodnar, G., Wong, F., 2003. Estimating exchange rate exposures: issues in model structure. Financial Management 32, 35-67.

Bollerslev, T., 1990. Modelling the coherence in short-run nominal exchange rates: a multivariate generalized ARCH model. The Review of Economics and Statistics 72, 498-505.

Booth, L., 1996. On the nature of foreign exchange exposure. Journal of Multinational Financial Management 6, $1-24$.

Boudoukh, J., Richardson, M., Whitelaw, R., 2006. The myth of long-horizon predictability. Review of Financial Studies 21, 1577-1605.

Brandt, M., Wang, K., 2003. Time-varying risk aversion and unexpected inflation. Journal of Monetary Economics 50, 1457-1498.

Carhart, M., 1997. On persistence in mutual fund performance. Journal of Finance 52, 57-82.

Chen, Y., 2011. Derivatives use and risk taking: evidence from the hedge fund industry. Journal of Financial and Quantitative Analysis 46, 1073-1106.

Chkili, W., Aloui, C., Nguyen, D., 2012. Asymmetric effects and long memory in dynamic volatility relationships between stock returns and exchange rates. Journal of International Financial Markets, Institutions and Money 22, 738-757.

Choi, J., Prasad, A., 1995. Exchange risk sensitivity and its determinants: a firm and industry analysis of U.S. multinationals. Financial Management 24, 77-88.

Chow, E., Lee, W., Solt, M., 1997a. The exchange rate exposure of US multinational firms. Journal of Business 70, 105-123.

Chow, E., Lee, W., Solt, M., 1997b. The economic exposure of U.S. multinational firms. Journal of Financial Research 20, 191-210.

Cornaggia, J., 2013. Does risk management matter? Evidence from the U.S. agricultural industry. Journal of Financial Economics 109, 419-440.

Dolde, W., 1993. The trajectory of corporate financial risk management. Journal of Applied Corporate Finance 6, 33-41.

Dominguez, K., Tesar, L., 2006. Exchange rate exposure. Journal of International Economics 68, 188- 218. 
Du, D., Hu, O., 2012. Foreign exchange volatility and stock returns. Journal of International Financial Markets, Institutions and Money 22, 1202-1216.

Efron, B., 1979. Bootstrap methods: another look at the jackknife. Annals of Statistics 7, 1-26.

Ehrhardt, M., 1991. Diversification and interest rate risk. Journal of Business Finance and Accounting 18, 43-59.

Faff, R., Marshall, A., 2005. International evidence on the determinants of foreign exchange rate exposure of multinational corporations. Journal of International Business Studies 36, 539-558.

Fama, E., French, K., 1992. The cross-section of expected stock returns. Journal of Finance 47, 427-465.

Fama, E., French, K., 1993. Common risk factors in the returns on stocks and bonds. Journal of Financial Economics 33, 3-56.

Fama, E., French, K., 2012. Size, value, and momentum in international stock returns. Journal of Financial Economics 105, 457-472.

Fehle, F., Tsyplakov, S., 2005. Dynamic risk management: theory and evidence. Journal of Financial Economics 78, 3-47.

Ferguson, M., Shockley, R., 2003. Equilibrium 'anomalies'. Journal of Finance 58, 2549-2580.

Ferson, W., Sakissian, S., Simin, T., 2003. Spurious regression in financial economics. Journal of Finance 58, 1393-1413.

Fleming, J., Kirby, C., Ostdiek, B., 1998. Information and volatility linkages in the stock, bond, and money markets. Journal of Financial Economics 49, 111-137.

Fraser, S., Pantzalis, C., 2004. Foreign exchange rate exposure of US multinational corporations: a firmspecific approach. Journal of Multinational Financial Management 14, 261-281.

Froot, K., Ramadorai, T., 2005. Currency returns, intrinsic value, and institutional-investor flows. Journal of Finance 60, 1535-1566.

Glosten, L., Jagannathan, R., Runkle, D., 1993. On the relation between the expected value and the volatility of the nominal excess return on stocks. Journal of Finance 48, 1779-1801.

He, J., Ng, L., 1998. The foreign exchange exposure of Japanese multinational corporations. Journal of Finance 53, 733-753.

Hutson, E., Stevenson, S., 2010. Openness, hedging incentives and foreign exchange exposure: a firm-level multi-country study. Journal of International Business 41, 105-122.

Jayasinghe, P., Tsui, A., 2008. Exchange rate exposure of sectoral returns and volatilities: evidence from Japanese industrial sectors. Japan and the World Economy 20, 639-660.

Jegadeesh, N., Titman, S., 1993. Returns to buying winners and selling losers: implications for stock market efficiency. Journal of Finance 48, 65-91.

Jin, Y. Jorion, P., 2006. Firm value and hedging: evidence from U.S. oil and gas producers, Journal of Finance 61, 893-919.

Jorion, P., 1990. The exchange-rate exposure of U.S. multinationals. Journal of Business 63, 331-345.

Kanas, A., 2000. Volatility spillovers between stock returns and exchange rate changes. Journal of Business Finance and Accounting 27, 447-467.

Longin, F., Solnik, B., 2001. Extreme correlation and international equity markets. Journal of Finance 56, 649-676.

Marston, R., 2001. The effects of industry structure on economic exposure. Journal of International Money and Finance 20,149-164.

Miller, K., Reuer, J., 1998. Asymmetric corporate exposures to foreign exchange rate changes. Strategic Management Journal 19, 1183-1191.

Muller, A., Verschoor, W., 2006a. Asymmetric foreign exchange risk exposure: evidence from U.S. multinational firms. Journal of Empirical Finance 13, 495-518.

Muller, A., Verschoor, W., 2006b. European foreign exchange risk exposure. European Financial Management 12, 195-220.

Muller, A., Verschoor, W., 2008. The Latin American exchange exposure of U.S. multinationals. Journal of Multinational Financial Management 18, 112-130.

Nelson, D., 1990. Conditional heteroskedasticity in asset returns: a new approach. Econometrica 59, 347370. 
Pantzalis, C., Simkins, B., Laux, P., 2001. Operational hedges and the foreign exchange exposure of U.S. multinational corporations. Journal of International Business 32, 793-812.

Pérez-González, F., Yun, H., 2013. Risk management and firm value: evidence from weather derivatives. Journal of Finance 68, 2143-2176.

Petkova, R., 2006. Do the Fama-French factors proxy for innovations in predictive variables? Journal of Finance 61, 581-612.

Ross, S., 1989. Information and volatility: the non-arbitrage martingale approach to timing and resolution irrelevancy. Journal of Finance 44, 1-17.

Prasad, A., Rajan, M., 1995. The role of exchange and interest risk in equity valuation: a comparative study of international stock returns. Journal of Economics and Business 47, 457-472.

Shapiro, A., 1974. Exchange rate changes, inflation, and the value of the multinational corporation. Journal of Finance 30, 485-502.

Silvennoinen, A., Teräsvirta, T., 2005. Multivariate autoregressive conditional heteroskedasticity with smooth transitions in conditional correlations. SSE/EFI Working Paper Series in Economics and Finance: No. 577.

Simpson, M., Ramchander, S., 2008. An inquiry into the economic fundamentals of the Fama and French equity factors. Journal of Empirical Finance 15, 801-815. 
Table 1: Mean equation coefficient estimates for all firms under the constant conditional correlation

Panel A: Three-factor CAPM

\begin{tabular}{lrrrrrrrr} 
& \multicolumn{2}{c}{$\lambda_{1, F X} R_{F X, t-1}$} & \multicolumn{2}{c}{$\gamma_{1, f} R_{f, t-1}$} & $\xi_{1} D_{t}$ & $\delta_{1} h_{2, t}^{2}$ \\
\cline { 2 - 9 } & \multicolumn{1}{c}{$\operatorname{pos}(+)$} & neg (-) & $\operatorname{pos}(+)$ & $\operatorname{neg}(-)$ & $\operatorname{pos}(+)$ & $\operatorname{neg}(-)$ & $\operatorname{pos}(+)$ & neg (-) \\
\hline $\mathrm{N}$ & $476(50)$ & $740(154)$ & $475(98)$ & $741(154)$ & $486(56)$ & $730(117)$ & $896(175)$ & $320(24)$ \\
Mean & $0.1183^{\mathrm{a}}$ & $-0.1608^{\mathrm{a}}$ & $0.0204^{\mathrm{a}}$ & $-0.0196^{\mathrm{a}}$ & $0.6121^{\mathrm{a}}$ & $-1.1115^{\mathrm{a}}$ & $0.0173^{\mathrm{a}}$ & $-0.0128^{\mathrm{a}}$
\end{tabular}

\begin{tabular}{rrrr}
\multicolumn{2}{c}{$\lambda_{2, F X} R_{F X, t-1}$} & $\rho_{12}$ \\
\hline $\operatorname{pos}(+)$ & neg (-) & $\operatorname{pos}(+)$ & neg $(-)$ \\
\hline $1,197(1,002)$ & $19(12)$ & $618(244)$ & $598(302)$ \\
$0.0459^{\mathrm{a}}$ & $-0.3449^{\mathrm{a}}$ & $0.0377^{\mathrm{a}}$ & $-0.0558^{\mathrm{a}}$
\end{tabular}

Panel B: Six-factor CAPM

\begin{tabular}{|c|c|c|c|c|c|c|c|c|c|c|c|c|c|c|c|c|c|c|}
\hline & \multicolumn{2}{|c|}{$\lambda_{3, F X} R_{F X, t-1}$} & \multicolumn{2}{|c|}{$\gamma_{2, f} R_{f, t-1}$} & \multicolumn{3}{|c|}{$\xi_{2} D_{t}$} & $\delta_{2} h_{2, t}^{2}$ & \multicolumn{2}{|c|}{$\eta_{1} S M B_{t}$} & \multicolumn{2}{|c|}{$\eta_{2} H M L_{t}$} & \multicolumn{2}{|c|}{$\eta_{3} M_{O} M_{t}$} & \multicolumn{2}{|c|}{$\lambda_{4, F X} R_{F X, t-1}$} & \multicolumn{2}{|r|}{$\rho_{34}$} \\
\hline & $\operatorname{pos}(+)$ & neg (-) & pos $(+)$ & neg (-) & $\operatorname{pos}(+)$ & neg (-) & $\operatorname{pos}(+)$ & neg (-) & pos $(+)$ & neg $(-)$ & pos (+) & neg (-) & pos (+) & neg $(-)$ & $\operatorname{pos}(+)$ & neg $(-)$ & $\operatorname{pos}(+)$ & neg (-) \\
\hline $\mathrm{N}$ & $549(60)$ & 671(124) & $535(91)$ & $685(118)$ & $539(59)$ & 681(93) & $1,017(871)$ & 203(106) & $975(709)$ & 245(99) & $611(352)$ & $609(339)$ & $935(197)$ & 285(17) & $216(1,015)$ & $4(0)$ & 698(304) & $522(238)$ \\
\hline Mean & $0.1260^{\mathrm{a}}$ & $-0.1480^{\mathrm{a}}$ & $0.0182^{\mathrm{a}}$ & $-0.0182^{\mathrm{a}}$ & $0.6614^{\mathrm{a}}$ & $-1.0829^{\mathrm{a}}$ & $0.6074^{\mathrm{a}}$ & $-0.1473^{\mathrm{a}}$ & $0.4496^{\mathrm{a}}$ & $-0.2292^{\mathrm{a}}$ & $0.1619^{\mathrm{a}}$ & $-0.1725^{\mathrm{a}}$ & $0.0191^{\mathrm{a}}$ & $-0.0126^{\mathrm{a}}$ & $0.0460^{\mathrm{a}}$ & $-0.0102^{\mathrm{b}}$ & $0.0399^{\mathrm{a}}$ & $-0.0501^{\mathrm{a}}$ \\
\hline$U$-statistic & $\begin{array}{r}-1.32 \\
(-1.40)\end{array}$ & $\begin{array}{r}1.53 \\
(1.21)\end{array}$ & $\begin{array}{r}1.42 \\
(0.82)\end{array}$ & $\begin{array}{r}1.65 \\
(-0.53)\end{array}$ & $\begin{array}{r}-1.32 \\
\left(-2.39^{b}\right)\end{array}$ & $\begin{array}{r}0.86 \\
(-0.58)\end{array}$ & $\begin{array}{r}-37.8^{\mathrm{a}} \\
\left(-21.3^{\mathrm{a}}\right) \\
\end{array}$ & $\begin{array}{l}-17.7^{\mathrm{a}} \\
\left(6.89^{\mathrm{a}}\right)\end{array}$ & N/A & N/A & N/A & N/A & N/A & N/A & $-0.86(-1.00)$ & $\begin{array}{c}2.08^{\mathrm{b}} \\
(1.21)\end{array}$ & $\begin{array}{r}-1.50 \\
(-1.02)\end{array}$ & $\begin{array}{r}1.63^{\mathrm{c}} \\
(-0.07)\end{array}$ \\
\hline
\end{tabular}

${ }^{\mathrm{a}},{ }^{\mathrm{b}}$, and ${ }^{\mathrm{c}}$ denote statistical significance (two-tailed) at the $1 \%, 5 \%$, and $10 \%$ levels, respectively. $N=1,216$ firms under the three-factor CAPM whereas $N=1,220$ firms under

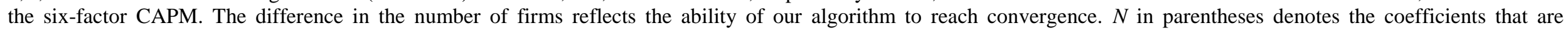

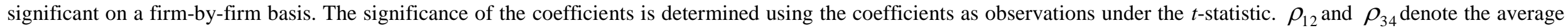
conditional correlation estimated using the conditional volatility of excess stock returns and FX rate changes in bivariate models that in each case contain the there- and six-factor CAPMs, respectively. $U$-statistic is the Mann-Whitney statistic for testing the null hypothesis that the coefficients across bivariate models are similar. The $U$-statistic in parentheses is for testing the null hypothesis that the coefficients that are significant firm-by-firm are also statistically different across bivariate models. All estimated coefficients are not shown to save space. The above estimates are for the bivariate GJR-GARCH-M under the assumption that the conditional correlations of the volatility of excess returns and FX rate changes (in the FX rate equation) are constant. The mean equations for the bivariate GJR-GARCH-M containing the three-factor CAPM for stock $i$ are:

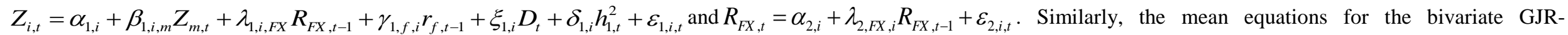
GARCH-M containing the six-factor CAPM for stock $i$ are:

$Z_{i, t}=\alpha_{3, i}+\beta_{2, i, m} Z_{m, t}+\lambda_{3, i, F X} R_{F X, t-1}+\gamma_{2, f, i} r_{f, t-1}+\xi_{2, i} D_{t}+\delta_{2, i} h_{2, t}^{2}+\eta_{1, i} S M B_{t}+\eta_{2, i} H M L_{t}+\eta_{3, i} M O M_{t}+\varepsilon_{3, i, t} \quad$ and $R_{F X, t}=\alpha_{i, 4}+\lambda_{4, F X, i} R_{F X, t-1}+\varepsilon_{4, i, t}$. In the table, we ignore the $i$ subscript since the coefficients are in averages. 
Table 2: Mean equation for the coefficient estimates of all firms by size, under the constant conditional correlation

Panel A: Three-factor CAPM

\begin{tabular}{|c|c|c|c|c|c|c|c|c|c|c|c|c|c|}
\hline & & \multicolumn{2}{|c|}{$\lambda_{1, F X} R_{F X, t-1}$} & \multicolumn{2}{|c|}{$\gamma_{1, f} R_{f, t-1}$} & \multicolumn{2}{|c|}{$\xi_{1} D_{t}$} & \multicolumn{2}{|c|}{$\delta_{1} h_{2, t}^{2}$} & \multicolumn{2}{|c|}{$\lambda_{2, F X} R_{F X, t-1}$} & \multicolumn{2}{|c|}{$\rho_{12}$} \\
\hline & & pos (+) & neg (-) & pos (+) & neg (-) & pos (+) & neg (-) & pos (+) & neg (-) & pos (+) & neg (-) & pos (+) & neg (-) \\
\hline \multirow[t]{2}{*}{ SMALL } & $\mathrm{N}$ & $154(14)$ & $226(43)$ & $134(29)$ & $248(51)$ & $131(17)$ & $251(54)$ & $279(63)$ & 101(5) & $374(302)$ & $6(2)$ & $189(64)$ & 191(88) \\
\hline & Mean & $0.1384^{\mathrm{a}}$ & $-0.2072^{\mathrm{a}}$ & $0.0225^{\mathrm{a}}$ & $-0.0186^{\mathrm{a}}$ & $0.7752^{\mathrm{a}}$ & $-1.3759^{a}$ & $0.0147^{\mathrm{a}}$ & $-0.0102^{\mathrm{a}}$ & $0.0456^{\mathrm{a}}$ & -0.2252 & $0.034^{\mathrm{a}}$ & $-0.044^{\mathrm{a}}$ \\
\hline \multirow[t]{2}{*}{ MEDIUM } & $\mathrm{N}$ & $127(16)$ & $255(61)$ & $178(36)$ & 204(46) & $153(20)$ & $227(26)$ & $279(53)$ & $101(7) 0$ & $374(324)$ & $6(4)$ & 181(67) & 201(93) \\
\hline & Mean & $0.1012^{\mathrm{a}}$ & $-0.1564^{a}$ & $0.0186^{\mathrm{a}}$ & $-0.0211^{\mathrm{a}}$ & $0.6008^{\mathrm{a}}$ & $-1.0871^{a}$ & $0.0178^{\mathrm{a}}$ & $-0.0130^{\mathrm{a}}$ & $0.0465^{\mathrm{a}}$ & $-0.3514^{c}$ & $0.035^{\mathrm{a}}$ & $-0.053^{\mathrm{a}}$ \\
\hline \multirow[t]{2}{*}{ LARGE } & $\mathrm{N}$ & $164(15)$ & $219(40)$ & $137(28)$ & $244(52)$ & 179(17) & 204(26) & 278(47) & $103(6)$ & $380(330)$ & $1(0)$ & 211(98) & $170(101)$ \\
\hline & Mean & $0.1079^{\mathrm{a}}$ & $-0.1208^{a}$ & $0.0260^{\mathrm{a}}$ & $-0.0197^{\mathrm{a}}$ & $0.5124^{\mathrm{a}}$ & $-0.8269^{a}$ & $0.0192^{\mathrm{a}}$ & $-0.0146^{\mathrm{a}}$ & $0.0458^{\mathrm{a}}$ & -0.8475 & $0.043^{\mathrm{a}}$ & $-0.075^{\mathrm{a}}$ \\
\hline
\end{tabular}

\begin{tabular}{|c|c|c|c|c|c|c|c|c|c|c|c|c|c|}
\hline & & \multicolumn{2}{|c|}{$\lambda_{3, F X} R_{F X, t-1}$} & \multicolumn{2}{|c|}{$\gamma_{2, f} R_{f, t-1}$} & \multicolumn{2}{|c|}{$\xi_{2} D_{t}$} & \multicolumn{2}{|c|}{$\delta_{2} h_{2, t}^{2}$} & \multicolumn{2}{|c|}{$\lambda_{4, F X} R_{F X, t-1}$} & \multicolumn{2}{|c|}{$\rho_{34}$} \\
\hline & & $\operatorname{pos}(+)$ & neg $(-)$ & $\operatorname{pos}(+)$ & neg (-) & $\operatorname{pos}(+)$ & neg (-) & $\operatorname{pos}(+)$ & neg (-) & $\operatorname{pos}(+)$ & neg (-) & $\operatorname{pos}(+)$ & neg (-) \\
\hline \multirow[t]{2}{*}{ SMALL } & $\mathrm{N}$ & $188(26)$ & 194(30) & $155(26)$ & $225(35)$ & $165(19)$ & $217(35)$ & $376(365)$ & $4(0)$ & 379(303) & $1(0)$ & 213(100) & $169(63)$ \\
\hline & Mean & $0.1454^{\mathrm{a}}$ & $-0.1916^{a}$ & $0.0193^{\mathrm{a}}$ & $-0.0172^{\mathrm{a}}$ & $0.7458^{\mathrm{a}}$ & $-1.3786^{\mathrm{a}}$ & $0.8190^{\mathrm{a}}$ & $-0.1038^{\mathrm{a}}$ & $0.0457^{\mathrm{a}}$ & -0.0121 & $0.040^{\mathrm{a}}$ & $-0.040^{\circ}$ \\
\hline \multirow[t]{2}{*}{ MEDIUM } & $\mathrm{N}$ & 151(12) & $231(46)$ & 193(33) & $189(30)$ & $169(23)$ & $213(26)$ & $358(310)$ & $24(5)$ & $381(327)$ & $0(0)$ & 213(81) & $169(77)$ \\
\hline & Mean & $0.1148^{\mathrm{a}}$ & $-0.1352^{a}$ & $0.0179^{\mathrm{a}}$ & $-0.0189^{\mathrm{a}}$ & $0.6865^{\mathrm{a}}$ & $-1.1426^{\mathrm{a}}$ & $0.5657^{\mathrm{a}}$ & $-0.0784^{a}$ & $0.0466^{\mathrm{a}}$ & N/A & $0.037^{\mathrm{a}}$ & $-0.050^{\circ}$ \\
\hline \multirow[t]{2}{*}{ LARGE } & $\mathrm{N}$ & $178(14)$ & $205(36)$ & $157(28)$ & $224(45)$ & $178(15)$ & $205(23)$ & $218(142)$ & $165(98)$ & $382(332)$ & $0(0)$ & 232(107) & $151(81)$ \\
\hline & Mean & $0.1105^{\mathrm{a}}$ & $-0.1250^{\mathrm{a}}$ & $0.0169^{\mathrm{a}}$ & $-0.0183^{a}$ & $0.5798^{a}$ & $-0.7666^{\mathrm{a}}$ & $0.3168^{\mathrm{a}}$ & $-0.1630^{\mathrm{a}}$ & $0.0459^{\mathrm{a}}$ & N/A & $0.043^{\mathrm{a}}$ & $0.064^{2}$ \\
\hline
\end{tabular}

${ }^{\mathrm{a}}, \mathrm{b}$, and ${ }^{\mathrm{c}}$ denote statistical significance at the $1 \%, 5 \%$, and $10 \%$ levels, respectively. The significance of the coefficients is determined using the coefficients as observations under the $t$-statistic. The $N$ denotes the sample size. $N$ in parentheses denotes the number of coefficients that are significant on a firm-by-firm basis. The estimates are obtained from two separate bivariate GJR-GARCH-M under the assumption of constant conditional correlation using Eqs. (1a) to 2(b). $\rho_{12}$ and $\rho_{34}$ are the average conditional correlation in the bivariate models under the three- and six-factor CAPMs, respectively. Small, medium and large firms have average capitalization values ranging from $\$ 0.15$ million to $\$ 386$ million, $\$ 386$ million to $\$ 2.1$ billion, and $\$ 2.1$ billion to $\$ 110$ billion, respectively. 
Table 3: Firms with structural breaks in their excess returns and significant FX rate coefficients

\begin{tabular}{|c|c|c|c|c|c|c|}
\hline \multirow[b]{2}{*}{$d$} & \multicolumn{3}{|c|}{ Three-factor CAPM } & \multicolumn{3}{|c|}{ Six-factor CAPM } \\
\hline & $\mathrm{LM}_{\mathrm{cc}}$ & Sig. cases & Percentage (\%) & $\mathrm{LM}_{\mathrm{cc}}$ & Sig. cases & Percentage (\%) \\
\hline 1 DAY & 128 & 20 & 15.63 & 147 & 22 & 14.97 \\
\hline 2 DAYS & 153 & 34 & 22.22 & 242 & 55 & 22.73 \\
\hline 5 DAYS & 257 & 54 & 21.01 & 230 & 40 & 17.39 \\
\hline 8 DAYS & 116 & 12 & 10.34 & 381 & 93 & 24.41 \\
\hline 11 DAYS & 142 & 22 & 15.49 & 221 & 58 & 26.24 \\
\hline 14 DAYS & 145 & 26 & 17.93 & 287 & 62 & 21.60 \\
\hline 17 DAYS & 218 & 51 & 23.39 & 371 & 110 & 29.65 \\
\hline 20 DAYS & 244 & 37 & 15.16 & 379 & 101 & 26.65 \\
\hline 30 DAYS & 308 & 86 & 27.92 & 203 & 78 & 38.42 \\
\hline 40 DAYS & 566 & 162 & 28.62 & 292 & 110 & 37.67 \\
\hline
\end{tabular}

$d$ denotes the return horizon. $\mathrm{LM}_{\mathrm{cc}}$ denotes the number of firms with significant structural breaks in their excess returns at each return horizon. The sig. cases denote the number of firms with significant (positive and negative) FX rate coefficients at each return horizon. 
Table 4: Estimates of smooth transition conditional correlation coefficients for firms with structural breaks

\begin{tabular}{|c|c|c|c|c|c|c|c|c|c|c|c|c|c|c|c|c|c|c|c|c|}
\hline \multirow{2}{*}{$\begin{array}{l}d \\
+/-\end{array}$} & \multicolumn{2}{|c|}{$1 \mathrm{DAY}$} & \multicolumn{2}{|c|}{2 DAYS } & \multicolumn{2}{|c|}{5 DAYS } & \multicolumn{2}{|c|}{8 DAYS } & \multicolumn{2}{|c|}{11 DAYS } & \multicolumn{2}{|c|}{14 DAYS } & \multicolumn{2}{|c|}{17 DAYS } & \multicolumn{2}{|c|}{20 DAYS } & \multicolumn{2}{|c|}{30 DAYS } & \multicolumn{2}{|c|}{40 DAYS } \\
\hline & $\rho_{i}>0$ & $\rho_{i}<0$ & $\rho_{i}>0$ & $\rho_{i}<0$ & $\rho_{i}>0$ & $\rho_{i}<0$ & $\rho_{i}>0$ & $\rho_{i}<0$ & $\rho_{i}>0$ & $\rho_{i}<0$ & $\rho_{i}>0$ & $\rho_{i}<0$ & $\rho_{i}>0$ & $\rho_{i}<0$ & $\rho_{i}>0$ & $\rho_{i}<0$ & $\rho_{i}>0$ & $\rho_{i}<0$ & $\rho_{i}>0$ & $\rho_{i}<0$ \\
\hline \multicolumn{21}{|c|}{ Panel A: Conditional correlation under the three-factor CAPM } \\
\hline$N$ & 53 & 75 & 58 & 95 & 78 & 179 & 40 & 76 & 44 & 98 & 63 & 82 & 99 & 119 & 108 & 136 & 185 & 123 & 247 & 319 \\
\hline$\rho_{12 a}$ & -0.088 & 0.110 & -0.093 & 0.057 & -0.114 & 0.107 & -0.151 & 0.176 & -0.167 & 0.194 & -0.177 & 0.184 & -0.217 & 0.255 & -0.272 & 0.278 & -0.281 & 0.230 & -0.239 & 0.228 \\
\hline$\rho_{12 b}$ & 0.060 & -0.066 & 0.083 & -0.118 & 0.137 & -0.162 & 0.162 & -0.198 & 0.199 & -0.194 & 0.186 & -0.198 & 0.154 & -0.160 & 0.161 & -0.220 & 0.190 & -0.184 & 0.163 & -0.255 \\
\hline$c_{1}$ & -0.043 & 0.051 & 0.022 & 0.010 & -0.119 & 0.008 & -0.100 & -0.130 & -0.070 & -0.230 & -0.224 & -0.208 & -0.136 & -0.264 & -0.276 & -0.398 & -0.338 & -0.328 & -0.365 & -0.427 \\
\hline$g_{1}$ & 74 & 80 & 100 & 100 & 100 & 100 & 100 & 100 & 100 & 100 & 100 & 73 & 75 & 63 & 84 & 77 & 42 & 44 & 33 & 35 \\
\hline \multicolumn{21}{|c|}{ Panel B: Conditional correlation under the six-factor CAPM } \\
\hline$N$ & 71 & 76 & 87 & 155 & 78 & 152 & 182 & 199 & 85 & 136 & 139 & 148 & 164 & 207 & 164 & 215 & 102 & 101 & 150 & 142 \\
\hline$\rho_{34 a}$ & -0.152 & 0.166 & -0.056 & 0.060 & -0.114 & 0.125 & -0.120 & 0.143 & -0.139 & 0.204 & -0.164 & 0.137 & -0.148 & 0.188 & -0.219 & 0.253 & -0.229 & 0.218 & -0.281 & 0.224 \\
\hline$\rho_{34 b}$ & 0.127 & -0.170 & 0.100 & -0.104 & 0.139 & -0.129 & 0.122 & -0.135 & 0.168 & -0.140 & 0.157 & -0.179 & 0.181 & -0.139 & 0.205 & -0.199 & 0.250 & -0.250 & 0.242 & -0.289 \\
\hline$c_{2}$ & -0.048 & 0.055 & 0.044 & -0.002 & -0.146 & 0.011 & -0.057 & -0.122 & -0.089 & -0.243 & -0.249 & -0.138 & -0.150 & -0.251 & -0.203 & -0.281 & -0.234 & -0.305 & -0.349 & -0.490 \\
\hline$g_{2}$ & 100 & 100 & 100 & 100 & 100 & 100 & 100 & 100 & 100 & 100 & 100 & 100 & 98 & 100 & 89 & 76 & 60 & 45 & 36 & 41 \\
\hline
\end{tabular}

$d$ denotes the return horizons at $d=1$ to $d=40$ days. $\mathrm{N}$ is the number of firms with a break in their excess returns for each regime $\rho_{12 a}$ and $\rho_{12 b}$ under the bivariate model containing the three-factor CAPM. $\rho_{34 a}$ and $\rho_{34 b}$ denote the conditional correlations for the bivariate model containing the six-factor CAPM. The values shown are simple averages of the individual coefficients based on the entire subsample. The symbol $+/$ - refers to the sign of the conditional correlation. For any $\rho_{i}>0$ the conditional correlation is positive, whereas for any $\rho_{i}<0$ the conditional correlation is negative. $c_{1}$ and $c_{2}$ denote the location coefficients for each regime; and $g_{1}$ and $g_{2}$ denote the smoothness coefficient (see Eqs. (6) and (8)). $g_{1}$ and $g_{2}$ are each set to 100 as an indicative value when the estimated $g_{1}$ and $g_{2}$ are larger than 100 . The estimates are performed on all firms with a structural break in their excess returns. 
Figure 1: Bar charts showing significant coefficients in mean equations for constant conditional correlation case The bar charts represent the number of positive (+), negative (-) and the total number of significant coefficients. The return horizons are $d=1,2,5,8,11,14,17,20,30$ and 40 days. These significant coefficients are obtained from the threeand six-factor CAPMs based on the GJR-GARCH-M. The original sample for the three-factor CAPM is 1,216 U.S. firms, whereas, the original sample for the six-factor CAPM is 1,220 U.S. firms. Variation in the sample size reflects the ability of our algorithm to reach convergence. To save space, we do not present the frequencies for the CAPM betas $\left(\beta_{1}\right.$ and $\left.\beta_{2}\right)$, the financial crisis and FX rate alphas $\left(\alpha_{2}\right.$ and $\left.\alpha_{4}\right)$ in the FX rate equations.

\section{Panel A: Three-factor CAPM}
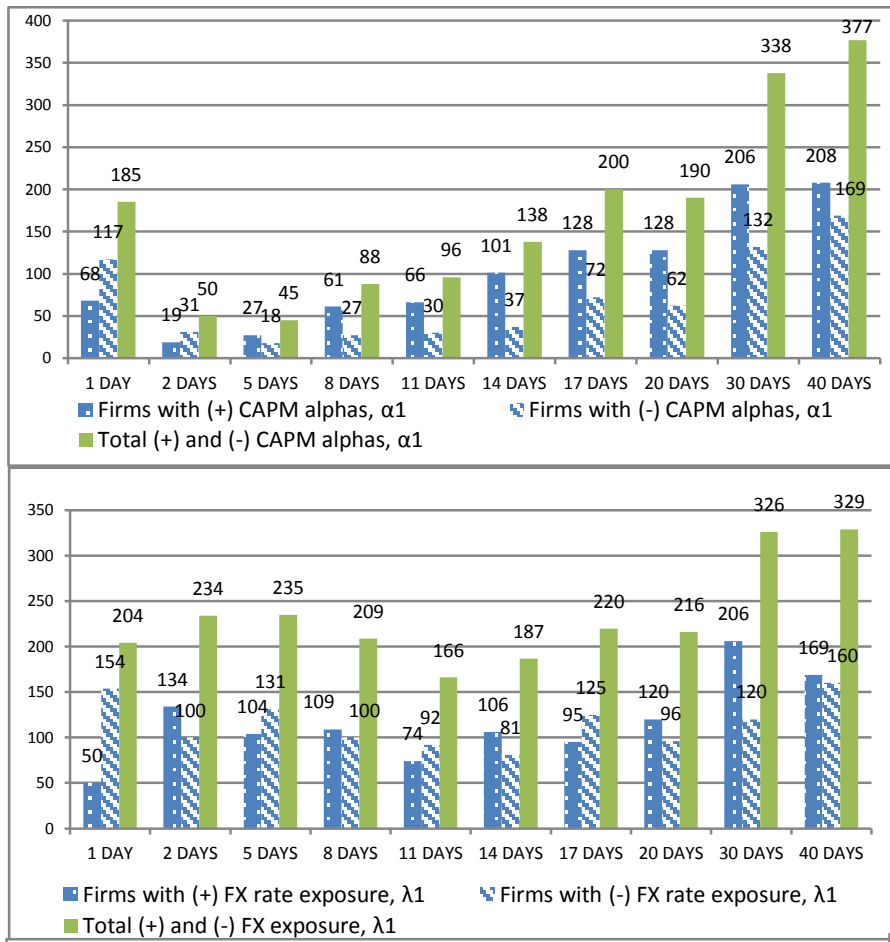

Total (+) and (-) FX exposure, $\lambda 1$

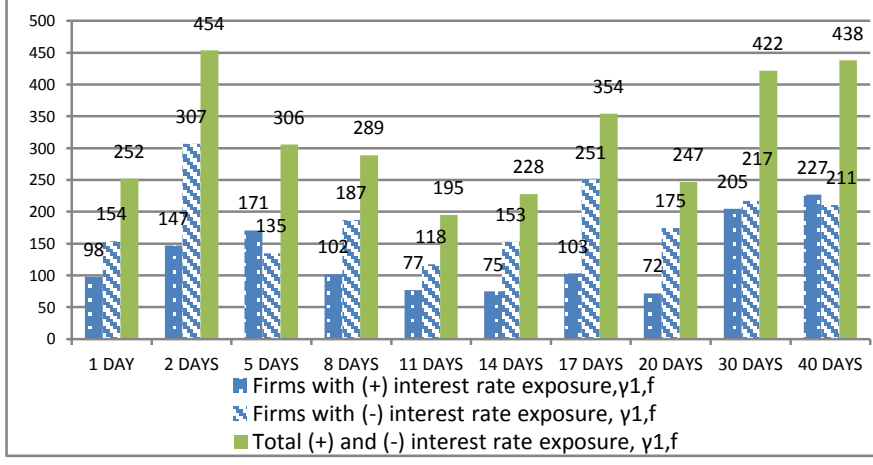

Panel B: Six-factor CAPM
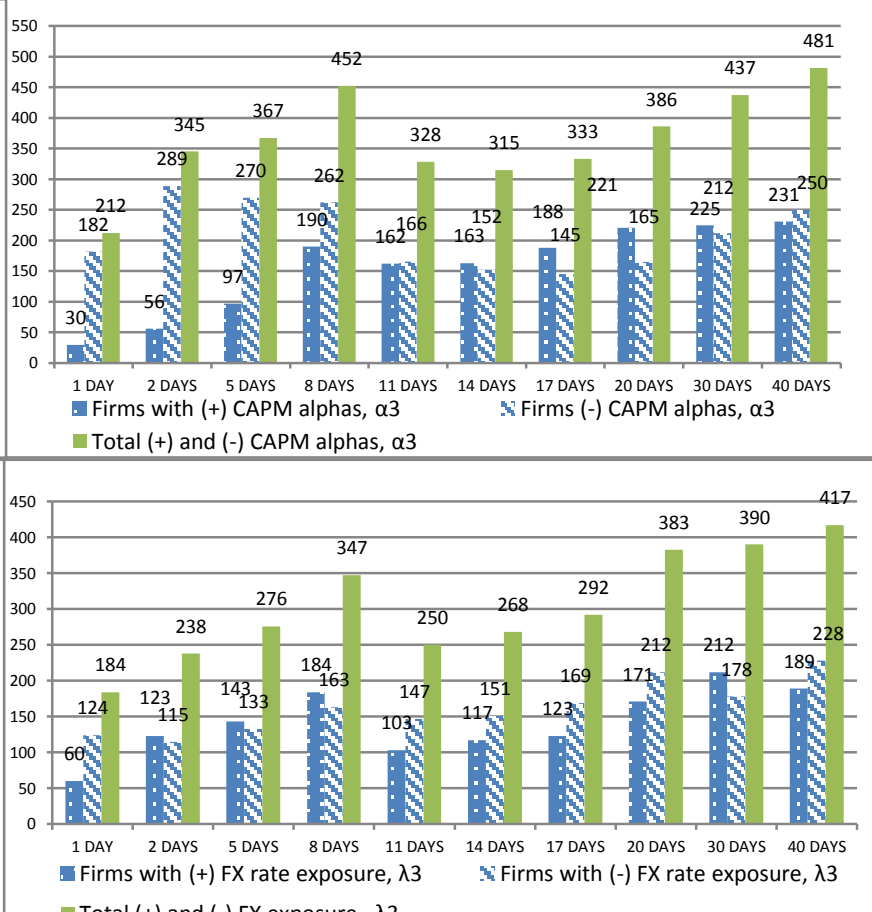

Total (+) and (-) FX exposure, $\lambda 3$

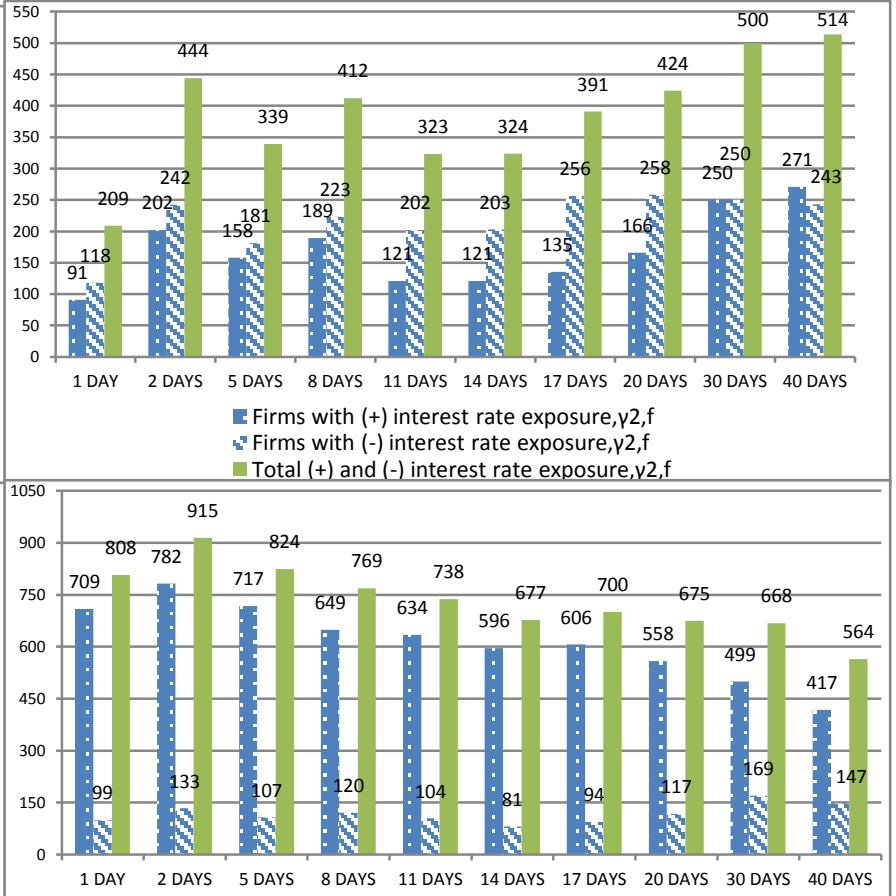

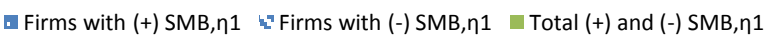

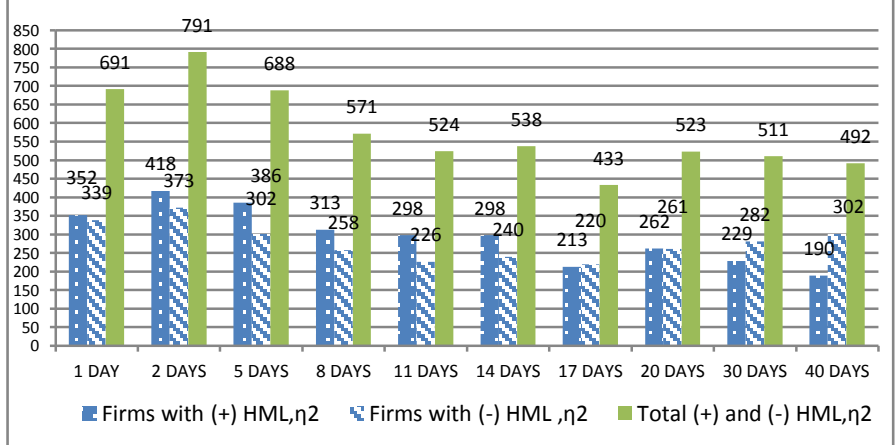


Figure 1: (Cont'd)

Panel B: Six-factor CAPM

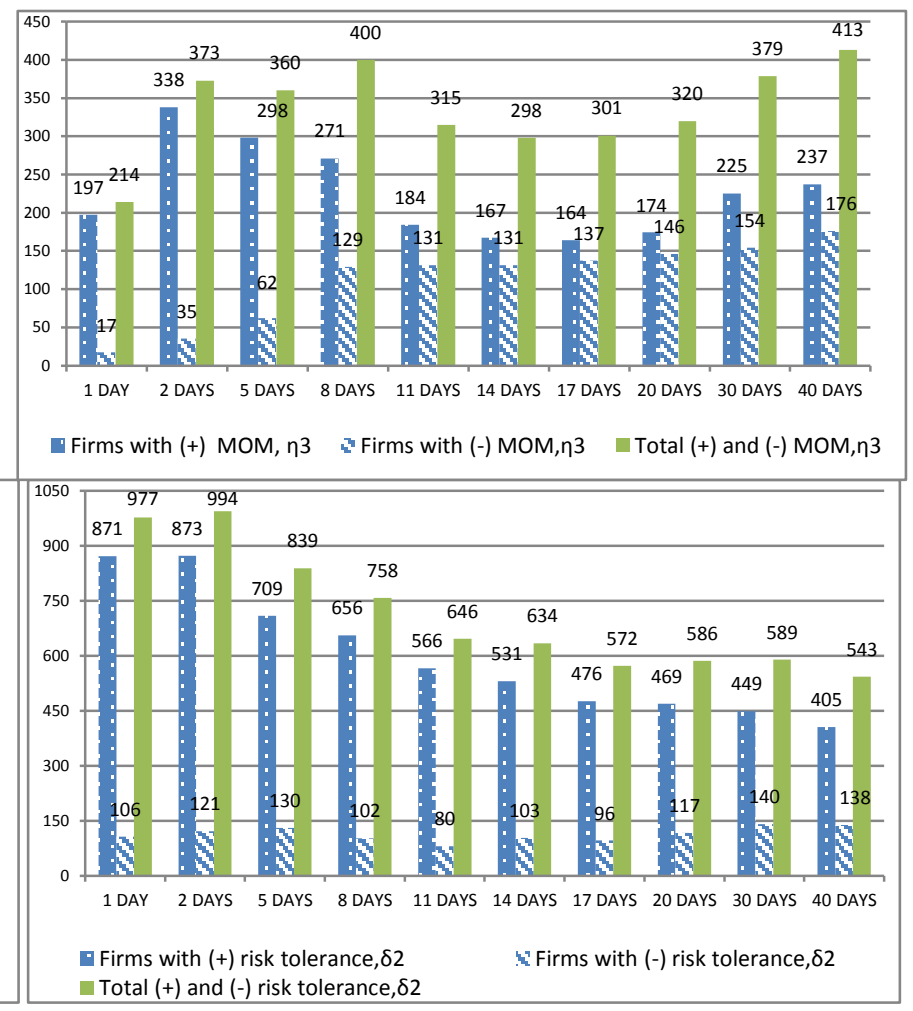

Panel C: Frequency of firms with positive and negative coefficients for exchange rate equations

$\begin{array}{ll}\text { i) Estimated jointly with three-factor CAPM } & \text { ii) Estimated jointly with six-factor CAPM }\end{array}$
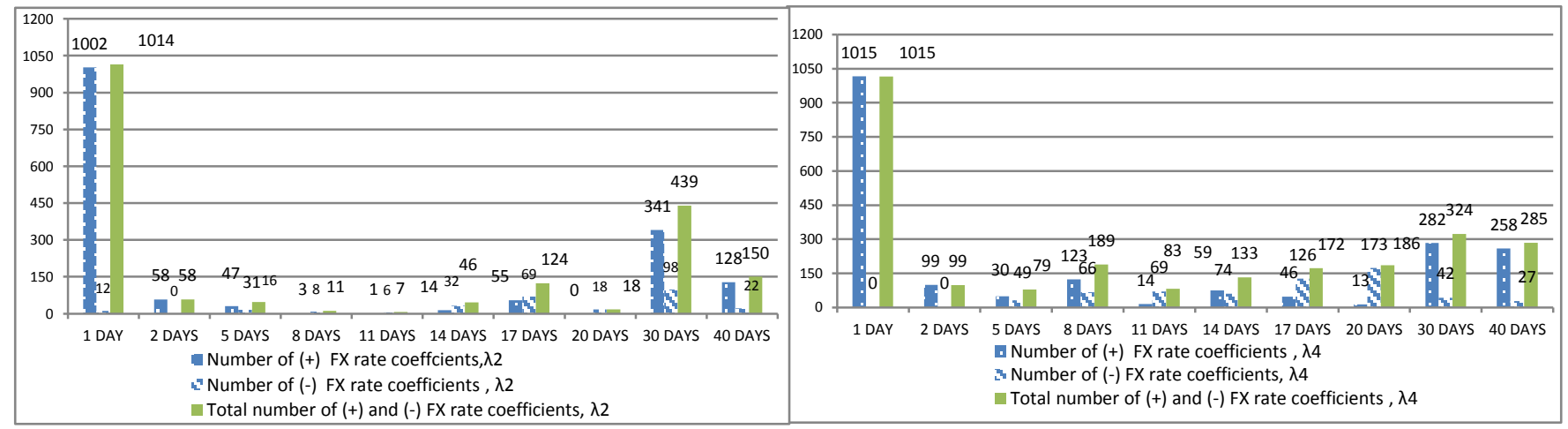

Panel D: Frequency of positive and negative conditional correlations

i) Estimated using conditional variance of three factor CAPM and FX rate equation ii) Estimated using conditional variance of six-factor CAPM and FX rate equation

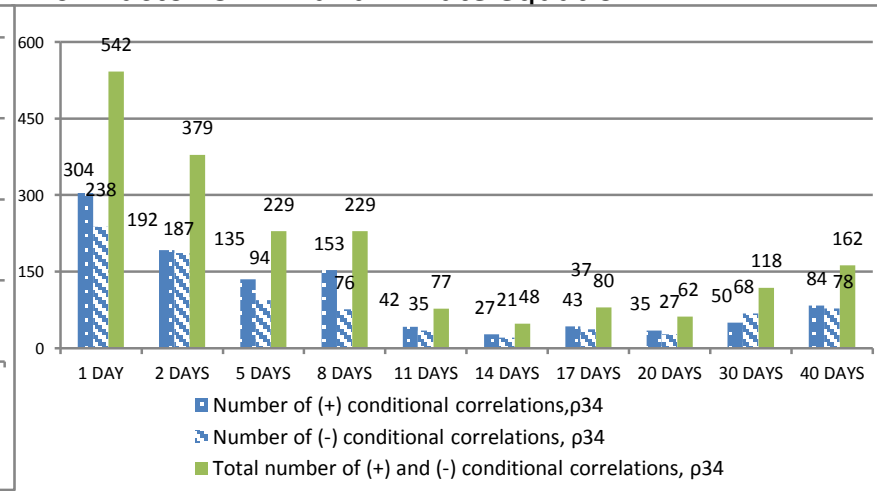


Figure 2: Bar charts for significant coefficients in mean equations under the smooth transition model The bar charts represent the number of positive (+), negative (-) and the total number of significant coefficients. The return horizons are $d=1,2,5,8,11,14,17,20,30$ and 40 days. These significant coefficients are obtained from the threeand six-factor CAPMs based on the STCC-GJR-GARCH-M. The original sample for the three-factor CAPM is 1,216 U.S. firms, whereas, the original sample for the six-factor CAPM is 1,220 U.S. firms. Variation in the sample size reflects the ability of our algorithm to reach convergence. To save space, we do not present the frequencies for the CAPM betas $\left(\beta_{1}\right.$ and $\left.\beta_{2}\right)$, the financial crisis and FX rate alphas $\left(\alpha_{2}\right.$ and $\left.\alpha_{4}\right)$ in the FX rate equations.

\section{Panel A:Three-factor CAPM Panel A:Six-factor CAPM}

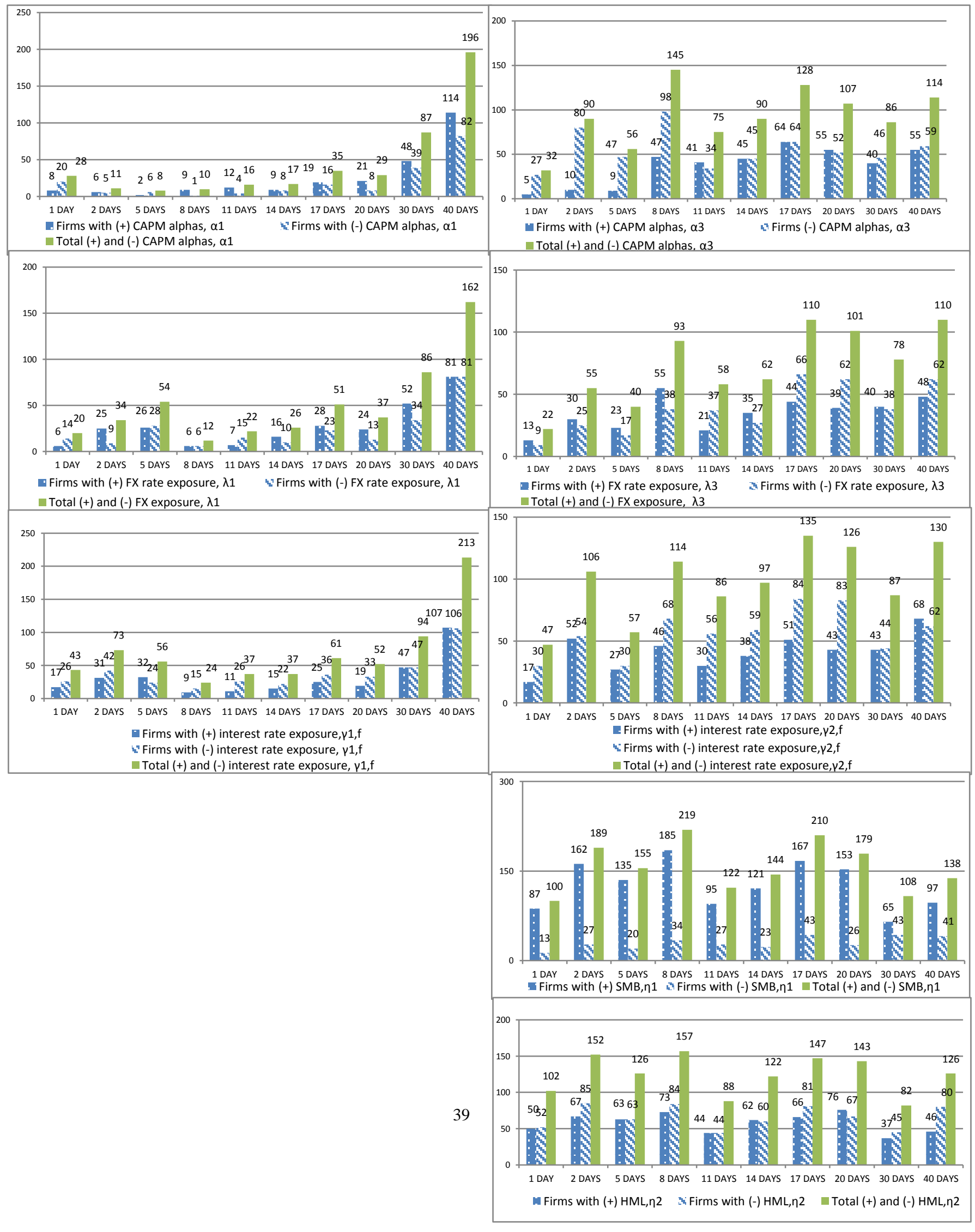


Figure 2: (Cont'd)

Panel A:Three-factor CAPM

Panel B: Six-factor CAPM

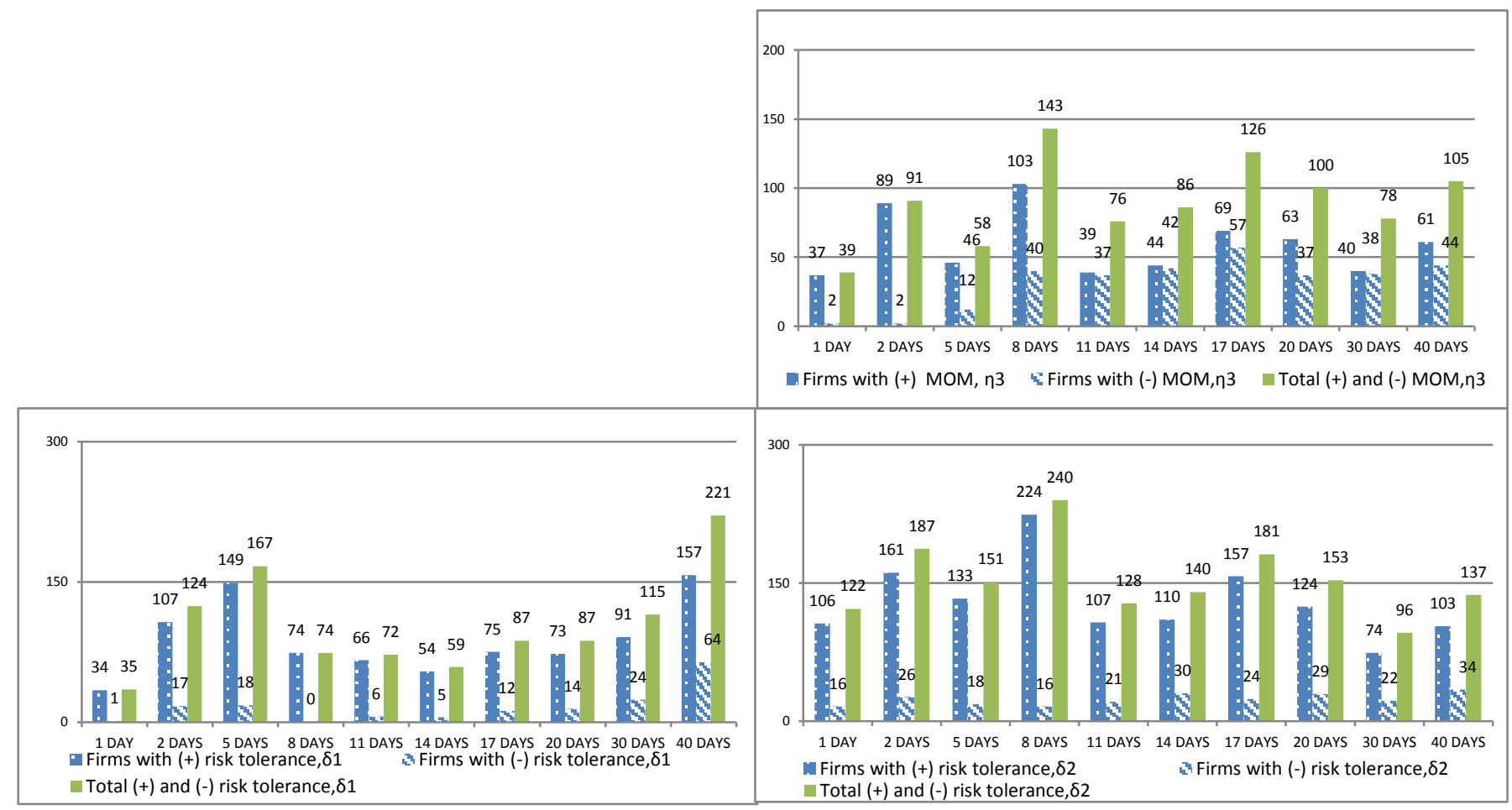

Panel C: Frequency of positive and negative coefficients for exchange rate equations

i)Estimated jointly with three-factor CAPM

ii) Estimated jointly with six-factor CAPM

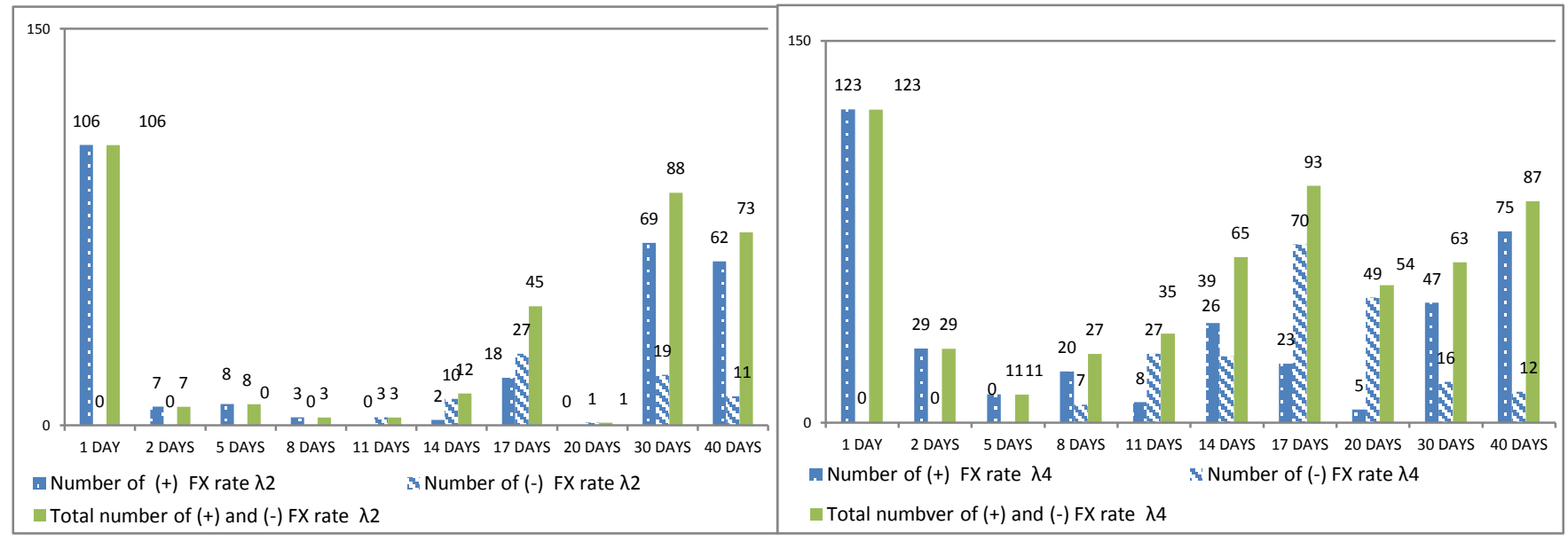




\section{Figure 3: Bar charts for significant coefficients and coefficients of transition function}

The bar charts represent the number of positive (+), negative (-) and the total number of significant coefficients. The return horizons are $d=1,2,5,8,11,14,17,20,30$ and 40 days. These significant coefficients are obtained from the three- and six-factor CAPMs based on the bivariate STCC-GJR-GARCH-M. The original sample for the three-factor CAPM is 1,216 U.S. firms, whereas, the original sample for the six-factor CAPM is1,220 U.S. firms. Variation in the sample size reflects the ability of our algorithm to reach convergence.

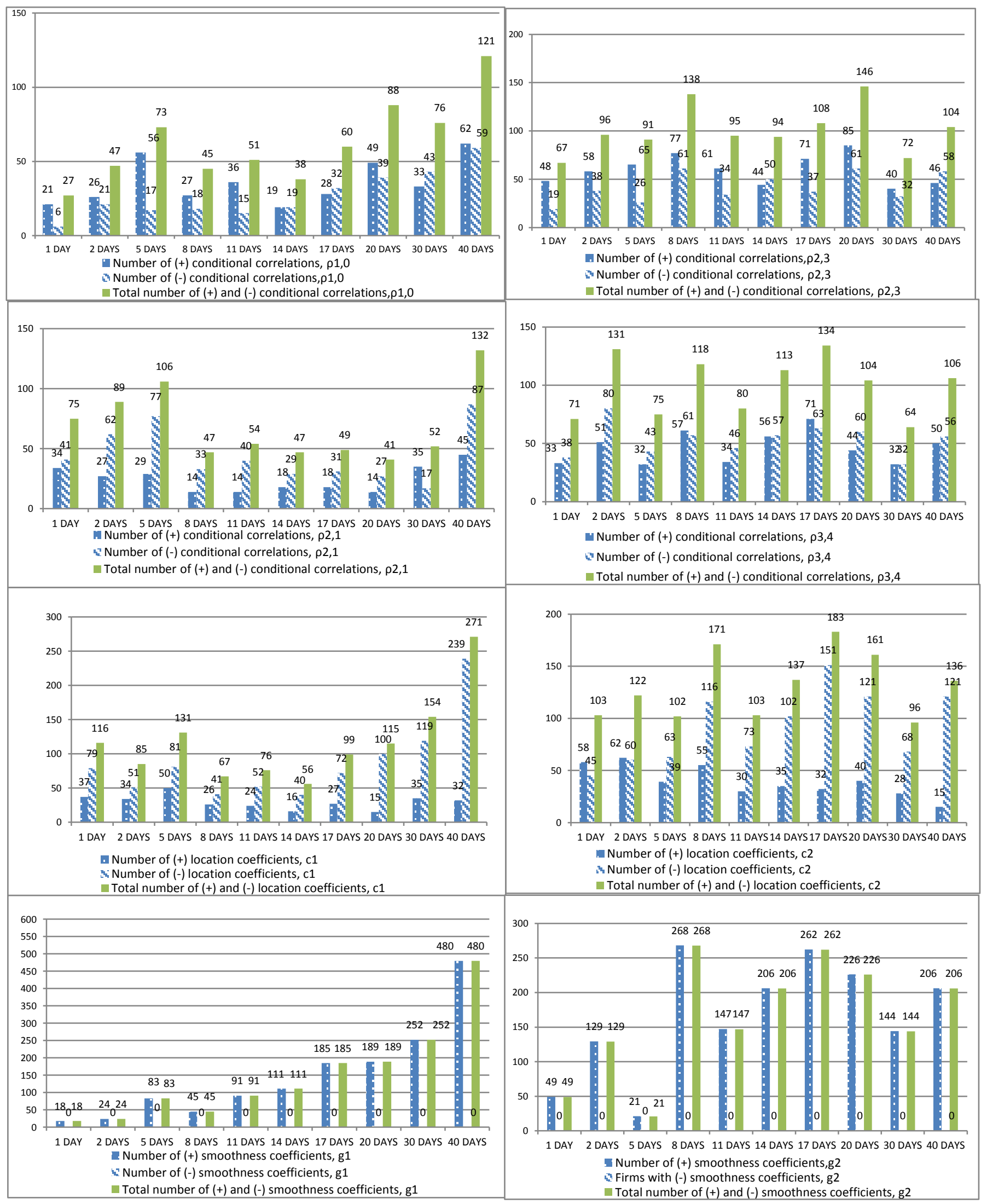


Figure 4: Bar charts for significant coefficients in variance equations under constant conditional correlation The bar charts represent the number of positive (+), negative (-) and the total number of significant coefficients. The return horizons are $d=1,2,5,8,11,14,17,20,30$ and 40 days. These significant coefficients are obtained from variance equations for the three- and six-factor CAPMs. The estimates are based on the GJR-GARCH-M. The original sample comprises of 1,216 U.S. firms under the three-factor CAPM and 1,220 U.S. firms under the sixactor CAPM. Variation in the sample size reflects the ability of our algorithm to reach convergence.

Panel A: Three-factor CAPM

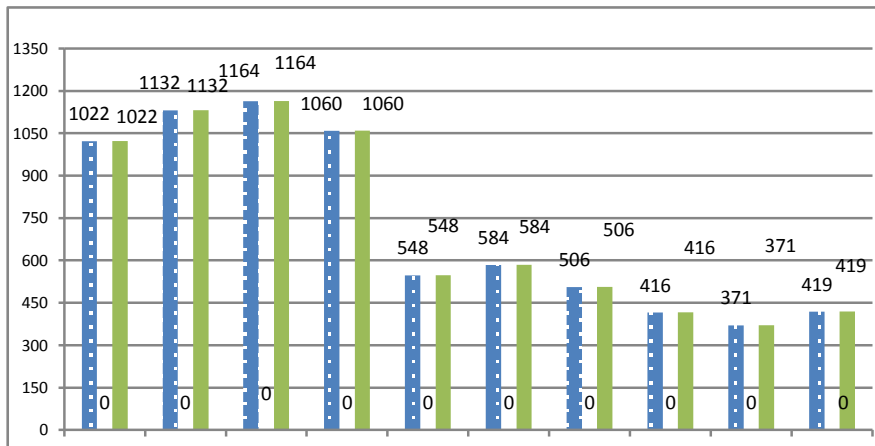

1 DAY 2 DAYS 5 DAYS 8 DAYS 11 DAYS 14 DAYS 17 DAYS 20 DAYS 30 DAYS 40 DAYS Firms with $(+)$ long-term variance, $\mu 1 \quad \Delta$ Firms with (-) long-term variance, $\mu 1$ - Total (+) and (-) long-trem variance, $\mu 1$

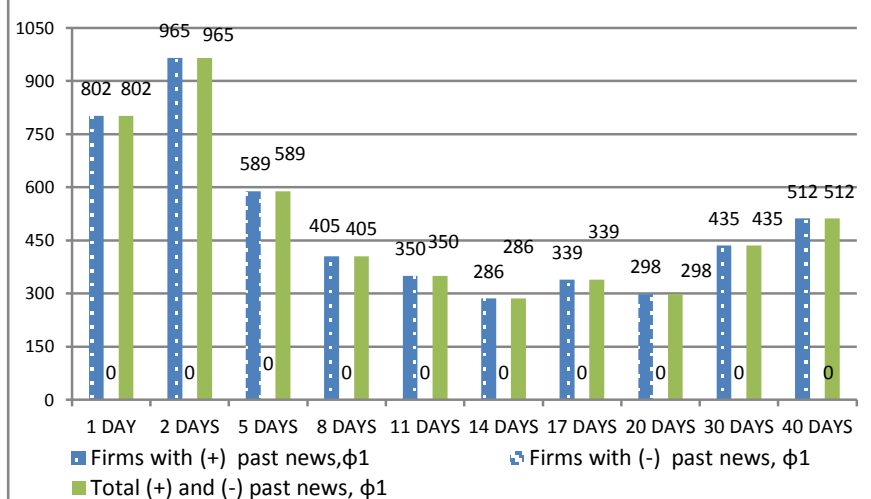

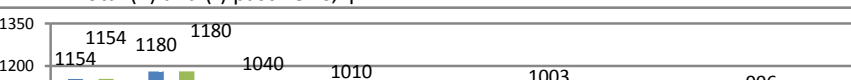

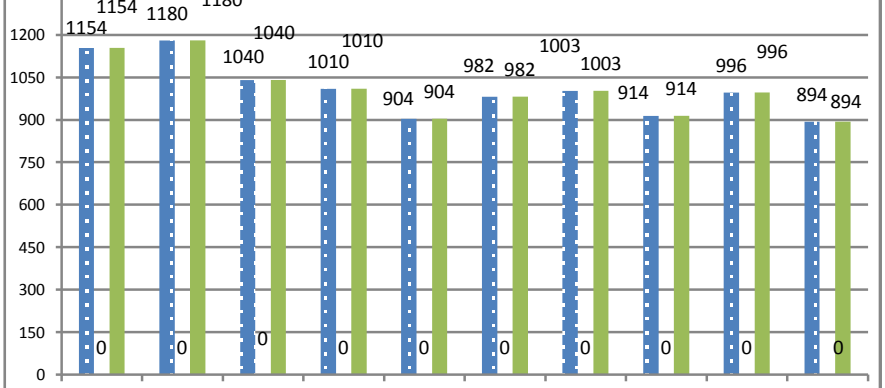

1 DAY 2 DAYS 5 DAYS 8 DAYS 11 DAYS 14 DAYS 17 DAYS 20 DAYS 30 DAYS 40 DAYS

글 Firms with $(+)$ past volatility, $\varphi 1$

Total $(+)$ and (-) past volatility, $\varphi 1$

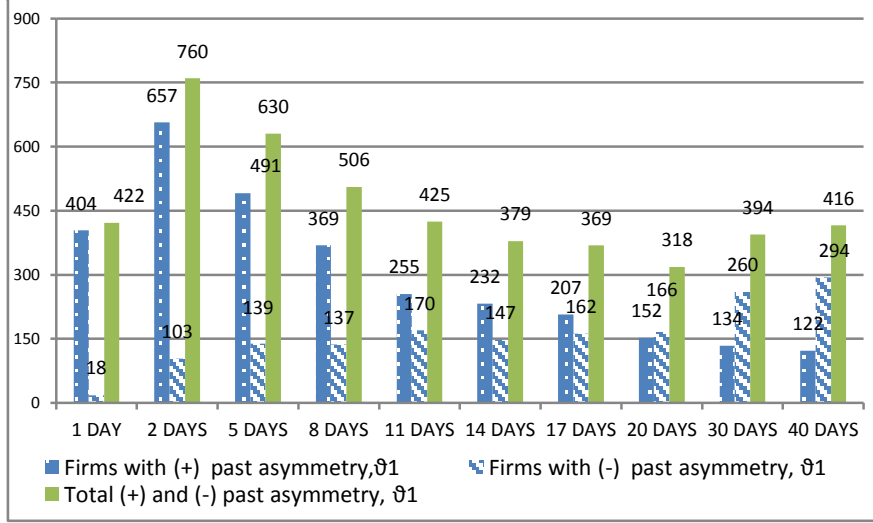

Panel B: Six-factor CAPMs

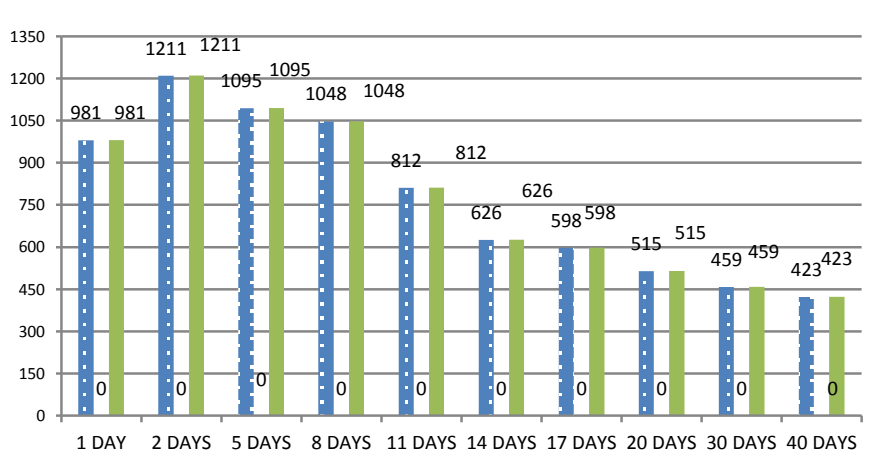

- Firms with $(+)$ long-term variance, $\mu 2 \quad \mathrm{~N}$ Firms with (-) long-term variance, $\mu 2$

- Total (+) and (-) long-term variance, $\mu 2$
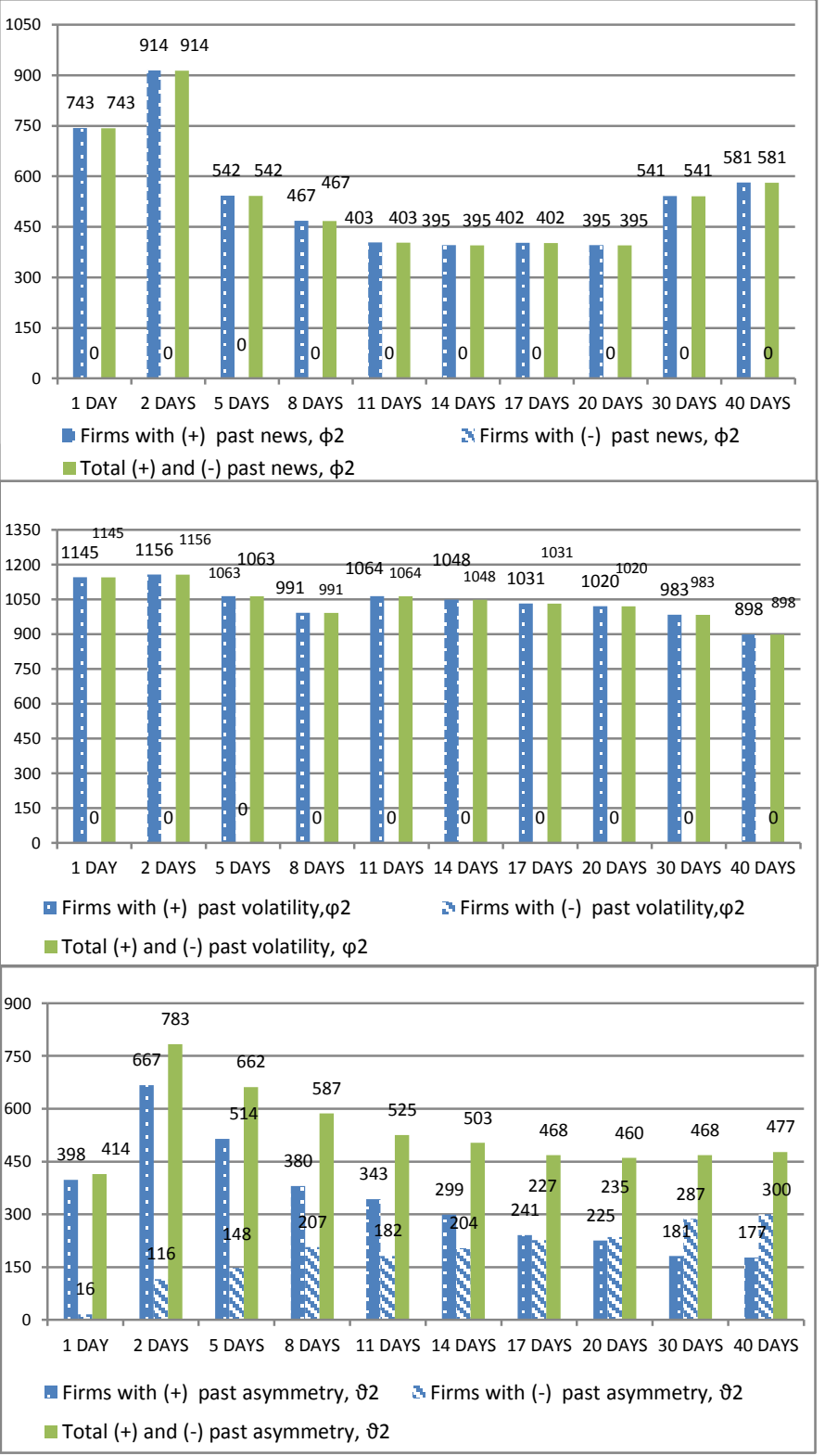
Figure 4: (Cont'd)

Panel C: Frequency of positive and negative coefficients for exchange rate equations i)Estimated jointly with three-factor CAPM

ii) Estimated jointly with six-factor CAPM

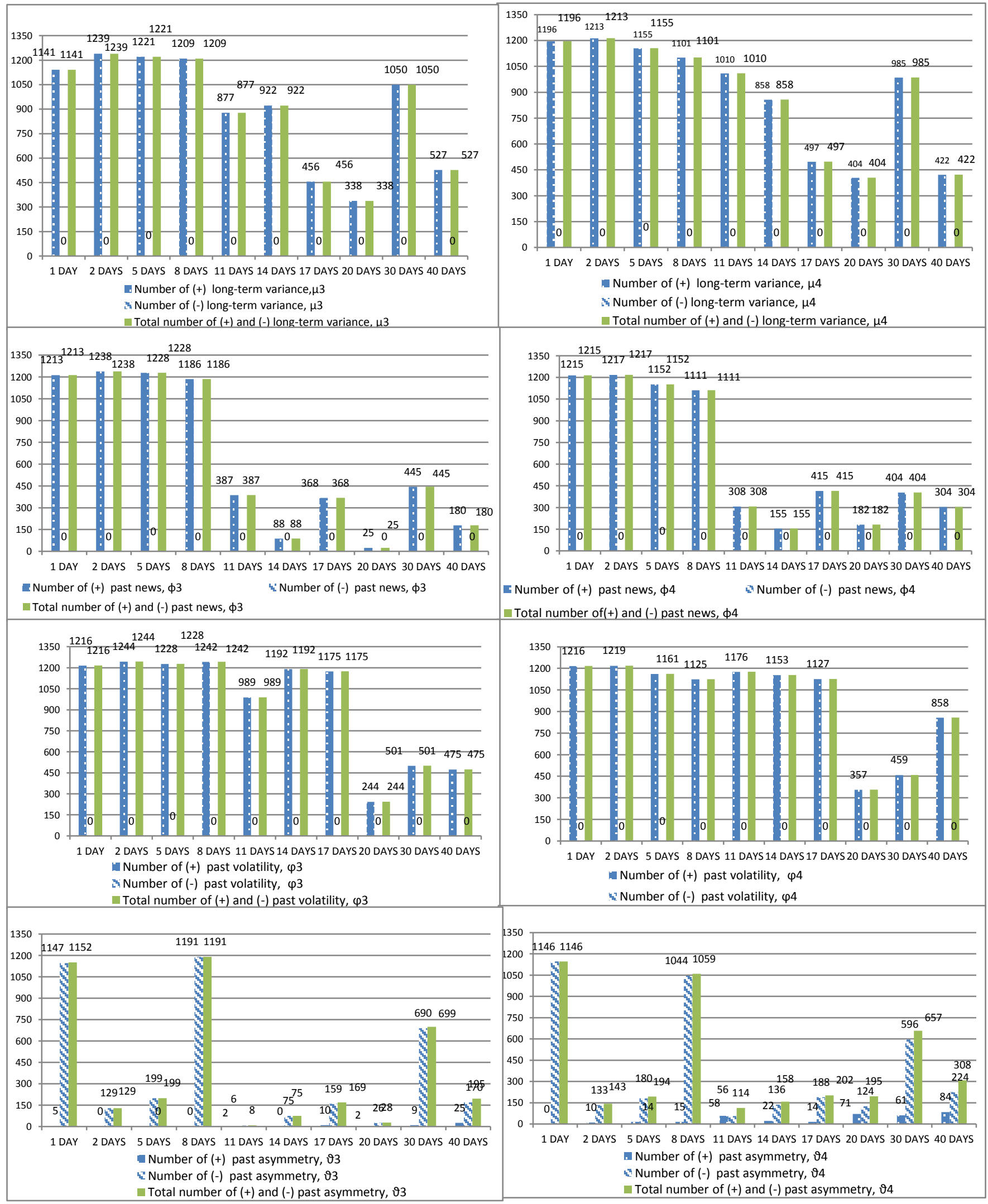

